\title{
Review Article \\ Heart Failure in Patients with Chronic Kidney Disease: A Systematic Integrative Review
}

\author{
Liviu Segall, Ionut Nistor, and Adrian Covic \\ Nephrology Department, Faculty of Medicine, University of Medicine and Pharmacy “Gr. T. Popa”, Strada. Universităţii No. 16, \\ 700115 Iaşi, Romania \\ Correspondence should be addressed to Liviu Segall; 1_segall@yahoo.com
}

Received 5 February 2014; Revised 21 April 2014; Accepted 22 April 2014; Published 15 May 2014

Academic Editor: Mitja Lainscak

Copyright (C) 2014 Liviu Segall et al. This is an open access article distributed under the Creative Commons Attribution License, which permits unrestricted use, distribution, and reproduction in any medium, provided the original work is properly cited.

Introduction. Heart failure (HF) is highly prevalent in patients with chronic kidney disease (CKD) and end-stage renal disease (ESRD) and is strongly associated with mortality in these patients. However, the treatment of HF in this population is largely unclear. Study Design. We conducted a systematic integrative review of the literature to assess the current evidence of HF treatment in CKD patients, searching electronic databases in April 2014. Synthesis used narrative methods. Setting and Population. We focused on adults with a primary diagnosis of CKD and HF. Selection Criteria for Studies. We included studies of any design, quantitative or qualitative. Interventions. HF treatment was defined as any formal means taken to improve the symptoms of HF and/or the heart structure and function abnormalities. Outcomes. Measures of all kinds were considered of interest. Results. Of 1,439 results returned by database searches, 79 articles met inclusion criteria. A further 23 relevant articles were identified by hand searching. Conclusions. Control of fluid overload, the use of beta-blockers and angiotensin-converting enzyme inhibitors or angiotensin receptor blockers, and optimization of dialysis appear to be the most important methods to treat HF in CKD and ESRD patients. Aldosterone antagonists and digitalis glycosides may additionally be considered; however, their use is associated with significant risks. The role of anemia correction, control of CKD-mineral and bone disorder, and cardiac resynchronization therapy are also discussed.

\section{Epidemiology}

During the past decade, the worldwide medical community has become increasingly aware of the fact that chronic kidney disease $(\mathrm{CKD})$ is a strong and independent risk factor for cardiovascular disease (CVD). In the US, for example, the prevalence of CVD in CKD patients reaches 63\%, in contrast with only $5.8 \%$ in people without CKD, and this prevalence is directly correlated with the severity of CKD [1]. In dialysisdependent end-stage renal disease (ESRD) patients, the risk of cardiovascular (CV) mortality is 10 -fold to 20 -fold higher than in age- and gender-matched control subjects without CKD $[2,3]$. This remarkable association of CKD with CVD is commonly explained by a typical clustering of several $\mathrm{CV}$ risk factors in patients with CKD; these factors may be classified as "traditional" (including advanced age, hypertension, diabetes, and dyslipidemia) and "nontraditional" (CKDspecific) ones (such as anemia, volume overload, mineral metabolism abnormalities, proteinuria, malnutrition, oxidative stress, and inflammation).

Heart failure (HF) is the leading CV complication in CKD patients and its prevalence increases with declining kidney function [4]. In the Atherosclerosis Risk in Communities (ARIC) study [5], a large, population-based study of US adults, the incidence of HF was 3-fold higher in individuals with an estimated glomerular filtration rate (GFR) $<60 \mathrm{~mL} / \mathrm{min} / 1.73 \mathrm{~m}^{2}$, compared with the reference group with an estimated GFR $\geq 90 \mathrm{~mL} / \mathrm{min} / 1.73 \mathrm{~m}^{2}$. In dialysis patients, the presence of $\mathrm{HF}$ at the start of dialysis is a strong and independent predictor of short-term [6] and longterm mortality, in both hemodialysis (HD) [7] and peritoneal dialysis (PD) patients [8]. The median survival of dialysis patients with baseline HF has been estimated to be 36 months, in contrast with 62 months for those without baseline HF [7]. Over $80 \%$ of ESRD patients who are recently diagnosed with 
$\mathrm{HF}$ are expected to die within only three years from the time of this diagnosis [9].

\section{Pathophysiology}

Abnormalities of left ventricular (LV) structure and function are very common in CKD and ESRD patients. Among ESRD patients, approximately $73.4 \%$ of those who are started on dialysis have LVH, 35.8\% have LV dilatation, and 14.8\% have LV systolic dysfunction [10]. Usually, LVH does not regress or even aggravates with time on dialysis and its presence is associated with a high risk of mortality and CV events, including sudden cardiac death [11].

Myocardial hypertrophy is associated with a reduction in the capillary density [12], which creates an imbalance between oxygen demands and supplies, thus causing ischemia [13]. Ischemia promotes myocardial cell apoptosis, as well as extracellular matrix and collagen accumulation, leading to interstitial fibrosis, which, in turn, induces LV stiffness, increased LV filling pressure, impaired diastolic filling, and diastolic dysfunction $[14,15]$. Moreover, myocardial fibrosis aggravates ischemia, by reduction of capillary density and coronary reserve [16], and considerably increases the risk of ventricular arrhythmias and sudden cardiac death [1719]. Associated coronary artery disease-also, very common in patients with $\mathrm{CKD}$ and ESRD-further contributes to ischemia, myocardial cell damage, and fibrosis [11].

From a hemodynamic view, LVH is an adaptive remodeling process of the LV, which compensates the increase in cardiac work induced by an increased afterload (pressure overload), an increased preload (volume overload), or both. Increased afterload may result from arterial hypertension, arterial stiffness, or valvular aortic stenosis and typically leads to a concentric thickening of the LV wall (concentric hypertrophy), which is meant to boost the intraventricular systolic pressure. Increased preload may be due to hypervolemia, anemia, and (in HD patients) high blood flow arteriovenous fistula; volume overload leads to the development of LV dilatation (eccentric LVH), by accumulation of new myocardial sarcomeres in series [20]. Afterload and preload factors often coexist in various degrees and combinations, with an additive or synergistic effect, which explains why both patterns, as well as a mixed pattern of $\mathrm{LVH}$, are commonly seen in CKD patients [21].

A large number of nonhemodynamic factors also contribute to the development of LVH and cardiomyopathy in CKD patients [12]. For example, hyperphosphatemia has been associated with high blood pressure (BP) [22], increased LV mass [23, 24], and diastolic dysfunction [25]. Excess angiotensin II can accumulate in the heart and promote myocyte hypertrophy, interstitial fibrosis, and microvascular disease, as well as cardiac conduction disturbances, QT prolongation, and arrhythmias [26]. High serum aldosterone, resulting from activation of renin-angiotensin system or other pathways, can induce myocardial fibrosis, possibly by release of transforming growth factor $\beta[17,27]$. Sympathetic overactivity, which has been demonstrated in CKD, is also deleterious to the heart and may induce LV concentric remodeling [28].

\section{Diagnosis}

$\mathrm{HF}$ is defined as a syndrome that can result from any structural or functional cardiac disorder that impairs the ability of the heart to function as a pump to support a physiological circulation [29]. HF may occur as a result of either systolic or diastolic dysfunction. The definition of $\mathrm{HF}$ requires the presence of symptoms, signs, and objective evidence of a structural or functional cardiac abnormality $[30]$.

3.1. Cardiac Imaging Studies. Two-dimensional (2D) echocardiography (including Doppler imaging) is a crucial investigation for the assessment of LV structure and function and for the diagnosis of causes of $\mathrm{HF}$, such as $\mathrm{LVH}$, myocardial ischemia, valvular disease, and pericardial effusion or constriction [31]. Echocardiography should provide measurements of ventricular diameters and volumes, wall thickness, chamber geometry, ejection fraction (EF), and regional wall motion abnormalities. In addition, atrial dimensions and/or volumes have to be measured. All valves must be evaluated for anatomic changes, flow abnormalities, and dysfunctions, such as valvular regurgitation. Combined quantification of mitral valve inflow pattern, pulmonary venous inflow pattern, and mitral annular velocity provides important information about the characteristics of LV filling and left atrial (LA) pressure [32]. Echocardiography has a key role in the diagnosis of systolic versus diastolic HF. Systolic HF is usually defined as $\mathrm{HF}$ with a $\mathrm{LVEF} \leq 50 \%[33,34]$. Diastolic HFalso referred to as $\mathrm{HF}$ with normal LVEF-requires three diagnostic criteria: (a) presence of signs and symptoms of HF, (b) presence of normal or mildly abnormal LVEF, and (c) evidence of diastolic LV dysfunction [35].

An echocardiogram is warranted in any CKD patient presenting with new cardiac symptoms or events [36]. For ESRD patients, the kidney disease outcomes quality initiative (KDOQI) guidelines recommend echocardiograms to be performed 1-3 months after the start of dialysis and every 3 years thereafter, regardless of symptoms [37]. Other authors even suggest a closer follow-up, with serial examinations every 12 to 18 months $[38,39]$.

CKD patients with significant LV systolic dysfunction should undergo an evaluation for coronary artery disease. This may include both noninvasive imaging (stress echocardiography, nuclear imaging, or computed tomographic angiography) and invasive imaging tests (coronary angiography), as recommended by KDOQI guidelines [37]. Coronary angiography is indicated in patients presenting with $\mathrm{HF}$ and known or suspected coronary artery disease, except for those ineligible for revascularization therapy. Instead, computed tomographic angiography may be used in cases with an intermediate likelihood of coronary artery disease; however, this procedure has potential technical limitations in CKD, because of the severity of vascular calcifications, which may reduce the accuracy of coronary 
imaging [31]. Cardiac magnetic resonance imaging (MRI) enables accurate measurements of LV and LA volume and can identify myocardial viability and scar tissue [40]; however, given the issues of cost and availability, as well as the risk of nephrogenic systemic fibrosis from gadolinium contrast in CKD patients, MRI is not currently recommended as a routine imaging test in this population [31].

3.2. Assessment of Fluid Status. Assessment of fluid status is very important in all CKD and, particularly, ESRD patients. In those with CKD and HF, volume overload may be both a result and a precipitating factor of the latter condition.

However, the best way to assess fluid status and dry weight in dialysis patients is still an unsolved issue. The ideal method should be highly sensitive and specific, readily available, inexpensive, fast and easy to use by clinicians, and capable of predicting clinical outcomes. Such a method still does not exist. Dry weight in ESRD patients is currently determined in most dialysis centers on a clinical basis, and it is commonly defined as the lowest body weight a patient can tolerate without developing intra- or interdialytic hypotension or other symptoms of dehydration [41, 42]. However, clinical findings have insufficient specificity, sensitivity, and objectivity; they are often contradictory and difficult to interpret, cannot detect small changes in hydration status, and cannot accurately predict the target-dry weight. Furthermore, this decades-old strategy has not contributed to reducing the CV mortality in ESRD patients, which is ultimately one of the main long-term goals of renal replacement therapy. Therefore, more objective and more sophisticated techniques of assessing volume status have been proposed, each of these having its own benefits and limitations.

The echocardiographic measurement of inferior vena cava diameter and collapsibility can accurately predict right atrial pressure and volume status in HD patients, and the adjustment of dry weight based on this technique was shown to prevent intradialytic adverse events, to reduce LV mass and LA size, and to improve quality of life $[43,44]$.

Relative plasma volume monitoring is a safe and inexpensive procedure that monitors relative plasma volume by analyzing blood density and allows automatic feedback control of the ultrafiltration rate that prevents relative plasma volume from reaching a critical level. This technique has decreased intradialytic hypotension [45], and a recent study suggested that it may aid dry weight assessment during HD [46]. However, there are no norms to guide fluid removal by this method and its benefit for clinical outcomes is not known $[47,48]$.

One of the most promising methods of assessing dry weight that have emerged in recent years is bioelectrical impedance analysis (BIA). This method estimates body composition, including total body water (TBW), extracellular water (ECW), and intracellular water (ICW), by measuring the body's resistance and reactance to electrical current. It has been validated in healthy subjects and various patient populations by isotope dilution and other body composition techniques [49]. The procedure is safe, simple, and relatively inexpensive. Several studies have proved the usefulness of BIA for the evaluation of dry weight in HD patients. Overhydration $>15 \%$ of ECW as measured by BIA was demonstrated to predict mortality [50]. MacHek et al. showed that the adjustment of fluid status guided by BIA led to significant reductions in systolic BP and antihypertensive medications in overhydrated HD patients and prevention of adverse events in underhydrated ones [51].

More recently, a novel method has been described that detects pulmonary congestion using ultrasound [52]. Although lung water excess was not directly related to hydration status assessed by BIA, lung water after dialysis showed a strong negative association with LVEF and positive association with LA volume and pulmonary pressure, clearly suggesting that chest ultrasonography may be reliable in detecting subclinical pulmonary congestion in dialysis patients.

3.3. Natriuretic Peptides. Atrial natriuretic peptide and brain natriuretic peptide (BNP) are produced by atrial and ventricular myocytes in response to an increase in atrial or ventricular diastolic filling pressure and wall distension [53, 54]. Myocardic cells are released in circulation pro-BNP, a precursor that is subsequently cleaved into the biologically active BNP and the biologically inactive NT-pro-BNP. Thus, plasma levels of BNP and NT-pro-BNP reflect LV wall stress [53]. These levels are greatly increased in patients with HF and they are strongly correlated with the severity of LV systolic and diastolic dysfunction $[53,55]$, as well as with the severity of HF, as assessed by the New York Heart Association (NYHA) classification [56, 57]. BNP and NT-pro-BNP are currently considered as useful biomarkers for the diagnosis and evaluation of HF in the general population $[58,59]$. They also have an important prognostic value, as independent predictors of mortality and other cardiac endpoints in patients with $\mathrm{HF}$ [60]. A recent systematic review of available data concluded that HF therapy guided by target-natriuretic peptide concentrations is associated with better outcomes [61].

In CKD and ESRD plasma levels of natriuretic peptides are affected by the impaired renal clearance [62]; however, they still maintain a strong relation with LV end-diastolic wall stress. In dialysis patients, plasma natriuretic peptides have shown significant associations with LVH [63], LV systolic and diastolic dysfunction [62, 64-66], and LA dilatation $[62,67]$. Furthermore, it has been demonstrated that BNP and NT-pro-BNP can predict the risk of HF in nondialysis CKD [63] and in PD patients [65], respectively. These data clearly suggest the potential interest in the use of natriuretic peptides in the diagnosis and management of HF in CKD and ESRD [68]. In these populations, however, there is still no evidence for the role of natriuretic peptides in the diagnosis of HF (either rule-in or rule-out), for their prognostic value, or for their utility in guiding the treatment of HF. Specific cutoff levels also need to be determined. 


\section{Treatment}

In this section, we discuss the therapy of chronic HF in patients with $\mathrm{CKD}$, looking up to the existing evidence in this population, as well as to relevant trials and guidelines in the general population with HF. However, we shall not refer to the prevention and treatment of various causes of HF (such as coronary artery disease or valvular heart disease) or to the management of complications like arrhythmias, sudden cardiac death, acute pulmonary edema, and cardiogenic shock, which are beyond the scope of this review.

4.1. Treatment Goals. The treatment of HF in patients with CKD is unclear, as there is very little strong evidence to support any recommendations. Guidelines for the management of HF in the general population may not apply entirely to those with CKD, since such patients (particularly those with severe renal impairment) were quite often excluded from most of the RCTs that served as a rationale for these guidelines. The paucity of specific evidence and recommendations may explain why CKD patients with HF are less likely to receive certain therapies that are commonly used in the general HF population [69]. Wang and Sanderson [69] pointed out that the main objectives of HF therapy in CKD (as well as in non-CKD) patients are the following: (1) to decrease the preload and afterload and to reduce $\mathrm{LVH}$, (2) to treat myocardial ischemia, and (3) to inhibit neurohumoral hyperactivity, especially the sympathetic nervous system and the renin-angiotensin-aldosterone system (RAAS).

\subsection{Treatment Options: A Systematic Review}

\subsubsection{Methods}

Eligibility Criteria. We conducted electronic searches in the Cochrane Central Register of Controlled Trials (Centralissue 4, 2014) and MEDLINE (1966 to April 2014), using search terms relevant to this review without language restriction (Table S1) see Supplementary Material available online at (http://dx.doi.org/10.1155/2014/937398). In addition, we hand-searched journals, conference proceedings, and current awareness alerts, without language restriction. Two reviewers independently screened the search results by title and abstract, and then full text, to identify eligible trials that fulfilled inclusion criteria. We considered all studies design that assessed any of the following interventions for the treatment of adults with heart failure and chronic kidney disease, as defined by the authors.

Types of Interventions. We considered comparisons including diuretics, beta-blockers, angiotensin-converting enzyme inhibitors, angiotensin receptor blockers, aldosterone antagonists, and digitalis glycosides. Optimization of dialysis, the role of anemia correction, control of CKD-mineral and bone disorder, and cardiac resynchronization therapy were also included.
Types of Outcome Measures. Primary outcomes were all-cause mortality and CV mortality. Measures of all other kinds were considered of interest.

\subsubsection{Results}

Study Selection. Of 1,439 results returned by database searches, 79 articles met inclusion criteria. A further 23 relevant articles were identified by hand searching. Figure 1 shows a summary of inclusion and exclusion algorithm.

4.2.3. Control of Fluid Overload. In CKD patients, maintaining salt and water balance and improving BP control are key strategies to reduce both the risk and the manifestations of $\mathrm{HF}$ [70]. The importance of this approach has been demonstrated particularly in dialysis patients, in whom rigorous limitation of salt intake and aggressive ultrafiltration were shown to prevent or reduce LV hypertrophy and dilatation, with little or no antihypertensive medication $[71,72]$.

Dietary salt restriction is fundamental for CKD patients with HF [12]; however, there are no randomized control trials (RCTs) to confirm its benefit on CV outcomes in this population. Diuretic therapy often requires higher doses than in HF patients with normal kidney function [12]. Loop diuretics should be used as first-line agents in patients with GFR $<30 \mathrm{~mL} / \mathrm{min} / 1.73 \mathrm{~m}^{2}$, because thiazides are relatively ineffective in these cases when used alone [73]. Resistance to loop diuretics is also common in patients with advanced CKD, because of glomerular loss, tubular resistance from chronic diuretic use, secondary hyperaldosteronism, reduced intestinal drug absorption, and inadequate salt and water intake [74]. The diuretic effect can be improved by increasing total daily doses or dosing frequency (to 3 times daily) or by using combination regimens (e.g., furosemide with metolazone or hydrochlorothiazide) [73]. Intravenous bolus administration or continuous infusion of diuretics may be used briefly in resistant cases. Possible side effects, such as hypovolemia, hypokalemia, and hyponatremia, require careful monitoring, particularly when high doses or combination therapy is used [73].

4.2.4. Treatment of Anemia. In non-CKD patients with HF, several studies have reported that treatment of anemia with erythropoiesis-stimulating agents (ESA) can improve functional status, quality of life [75, 76], and LVEF [77]. A metaanalysis of 7 RCTs including 650 patients [78] showed a lower risk of hospitalization for HF, with no difference in adverse events, but also no significant decrease in mortality with rHuEPO treatment.

In patients with CKD and ESRD, anemia has also been associated with LV hypertrophy and dilatation [79]. The presence of anemia during the first year of renal replacement therapy was associated with an increase in the prevalence of LVH [2]. In CKD, a few nonrandomized studies have shown a regression of $\mathrm{LVH}$ after correction of anemia $[80,81]$; however, prospective RCTs found no evidence that this therapy can improve CV outcomes [81]. An RCT by Foley et al. [82] failed to show regression of LVH after normalization 


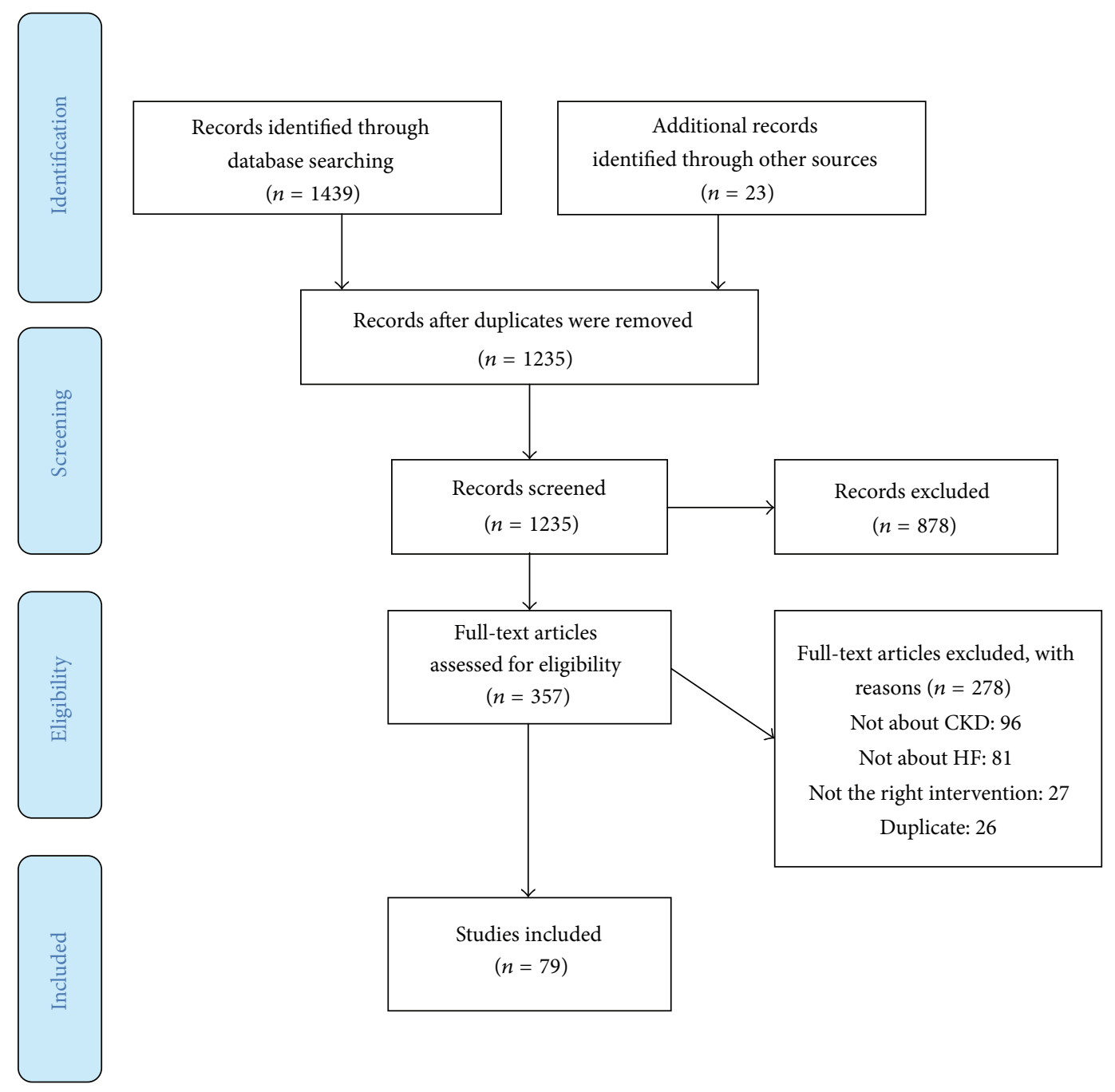

FIGURE 1: Inclusion and exclusion flow diagram.

of hemoglobin levels using rHuEPO in HD patients. Several subsequent large RCTs in patients with CKD and ESRD, including the Normal Hematocrit Trial [83], the CREATE (Cardiovascular Risk Reduction by Early Anemia Treatment with Epoetin Beta) study [84], the CHOIR (Correction of Hemoglobin and Outcomes in Renal Insufficiency) study [85], and the TREAT (Trial to Reduce Cardiovascular Events with Aranesp Therapy) study [86], showed an increased risk of death and $\mathrm{CV}$ events with erythropoiesis-stimulating agents. Although correction of anemia may improve myocardial oxygen delivery, it is thought that increased blood viscosity and BP associated with rHuEPO therapy could explain the adverse CV effects [87]. Considering these data, target serum hemoglobin of 11 to $12 \mathrm{~g} / \mathrm{dL}$ has been recommended for CKD patients by the Anaemia Working Group of the European Renal Best Practice [88]. However, no RCT has specifically addressed the optimal hemoglobin level in CKD patients with HF, so far. Therefore, it seems reasonable, for now, to treat these patients similarly to the general CKD population, avoiding the complete correction of anemia [89].
4.2.5. Management of CKD-Mineral and Bone Disorder. Achieving adequate phosphate, calcium, vitamin $\mathrm{D}$, and parathyroid hormone (PTH) levels is a reasonable treatment goal in CKD patients, with or without heart disease, although their benefits for preventing or improving $\mathrm{HF}$ in these patients are uncertain.

Hyperphosphatemia can promote LVH, possibly through changes in arterial stiffness, in systemic vascular resistance or through direct myocardial effects [23-25, 90]. However, there are yet no RCTs showing that a therapeutic decrease in serum phosphorus can prevent or reduce LVH or LV dysfunction in CKD patients. The use of non-calcium-containing phosphate binders is preferable, according to KDIGO guidelines [91], although a recent systematic review did not find sufficient evidence to confirm the superiority of these agents over calcium-based binders for CV end-points [92].

Parathyroid hormone has long been regarded as a $\mathrm{CV}$ toxin in uremia, particularly on the account of experimental studies. It has recently been suggested that some of the possible cardiac adverse effects of PTH could be mediated 
by fibroblast growth factor-23 (FGF23), which has been shown to directly induce $\mathrm{LVH}$ in both in vitro and in vivo studies [93, 94]. In ESRD patients, very high PTH levels (e.g., $>500 \mathrm{pg} / \mathrm{mL}$ ) have been associated with persistent LVH $[69,95]$. However, there is still no evidence that therapeutic lowering of PTH might prevent or ameliorate $\mathrm{HF}$ in $\mathrm{CKD}$ patients.

In experimental studies, 25-OH-vitamin $\mathrm{D}$ deficiency has been associated with myocardial fibrosis and systolic dysfunction [96]. Low serum vitamin D also stimulates the RAAS, resulting in vasoconstriction and salt and water retention, which further promotes arterial stiffening [97]. In animal models, treatment with active vitamin $\mathrm{D}$ inhibits endothelin-induced myocyte hypertrophy [98], reduces LV mass, and improves LV function, in parallel with a decrease in plasma BNP and renin activity [99].

In CKD patients, vitamin $\mathrm{D}$ deficiency has also been associated with LV dysfunction and risk of CV events, including HF [100]. Treatment with intravenous calcitriol in a short-term uncontrolled study in HD patients with secondary hyperparathyroidism showed partial regression of LVH and a decrease in plasma renin activity and angiotensin II levels [101]. A very recent randomized study [102] compared the effect of alfacalcidol versus no treatment in 14 patients with CKD stage 4, secondary hyperparathyroidism, and LVH. After 6 months, in the alfacalcidol-treated patients the LV mass index remained stable, but the LV systolic function (shortening fraction) significantly increased, while PTH decreased by $72 \%$. Another recent but uncontrolled study [103] evaluated the effect of cholecalciferol supplementation on cardiac function in $30 \mathrm{HD}$ patients with low vitamin $\mathrm{D}$ and low PTH levels. After 6 months, the authors found a significant increase in serum $25(\mathrm{OH}) \mathrm{D}$ levels, but no changes in PTH and phosphate, whereas LV mass index was significantly reduced by $16 \mathrm{~g} / \mathrm{m}^{2}$.

Selective vitamin D receptor activators, such as paricalcitol, provide similar efficacy as vitamin D but are usually not associated with increases in serum concentrations of calcium and phosphorus. This explains the interest in these agents as a potential new approach for CVD in CKD patients [97]. However, the recent PRIMO study [104], a multinational, double-blind, randomized, placebo-controlled trial in 227 patients with stages 3 and $4 \mathrm{CKD}$, mild-to-moderate $\mathrm{LVH}$, and preserved LVEF, failed to demonstrate any significant effect of paricalcitol on LV mass and diastolic function. Therefore, the role of vitamin $\mathrm{D}$ receptor activators in the treatment of cardiomyopathy and $\mathrm{HF}$ in $\mathrm{CKD}$ patients still remains to be elucidated.

4.2.6. Beta-Blockers. Beta-blockers have been evaluated in more than 20,000 patients with HF in over 20 placebocontrolled clinical trials. These trials have almost unanimously proven that these drugs can alleviate symptoms, improve the NYHA class, increase the LVEF, reduce hospitalizations, and, most importantly, prolong survival. The benefits of beta-blockers have been confirmed in patients with or without coronary artery disease and with or without diabetes, as well as in women and black patients. Favorable results were also seen in patients already taking ACEIs, which suggests that combined blockade of the two neurohormonal systems may have additive effects [32].

Three beta-blockers have been found to reduce mortality in patients with HF: bisoprolol and sustained-release metoprolol, which selectively block beta-1-receptors, and carvedilol, which blocks alpha-1-, beta-1-, and beta-2receptors. According to the guidelines of the American College of Cardiology Foundation/American Heart Association, a beta-blocker (one of these three agents) should be prescribed to all patients with stable HF due to systolic dysfunction, unless contraindicated or not tolerated [32]. Common side effects of beta-blockers include fluid retention and worsening HF at initiation of therapy, fatigue, bradycardia, and hypotension, and prevention of these effects requires careful dosage titration and clinical monitoring.

In patients with CKD, sympathetic overactivity is thought to play an important role in the pathogenesis of hypertension [105] and LVH [106]. High plasma norepinephrine levels predict adverse $\mathrm{CV}$ events and mortality in dialysis patients [107]. However, there is still limited evidence about the efficacy and tolerability of beta-blockers in patients with $\mathrm{HF}$ and renal dysfunction, since RCTs in HF have most often excluded individuals with CKD [108].

Several observational studies [109-111] have reported promising results. The Cooperative Cardiovascular Project [110] was a nonrandomized observational study using propensity score matching in patients over 65 years who survived a myocardial infarction. In the 2613 participants on beta-blockers, a greater benefit was noted for patients with serum creatinine levels $>2.0 \mathrm{mg} / \mathrm{dL}$. In a retrospective cohort study of 2550 patients enrolled in the US Renal Data System (USRDS) Wave 2 [111], beta-blocker use was associated with a lower adjusted risk of $\mathrm{HF}$ and cardiac death in patients without a history of HF; however, no association was observed in those with previous HF.

In predialysis CKD patients, more convincing evidence has been provided by recent post hoc analyses of 3 RCTs using beta-blockers in patients with HF [112-114]. The Metoprolol CR/XL Controlled Randomized Intervention Trial in Chronic HF (MERIT-HF) studied the effect of metoprolol in comparison with placebo in 3991 patients with symptomatic systolic HF (NYHA classes II to IV and LVEF < 40\%). In a secondary analysis, patients were divided into 3 renal function subgroups: eGFR $>60(n=2496)$, eGFR 45 to $60(n=976)$, and eGFR $<45 \mathrm{~mL} / \mathrm{min} / 1.73 \mathrm{~m}^{2}(n=493)$. The beneficial effect of metoprolol on clinical outcomes was significant and similar across all subgroups. In patients with eGFR <45, beta-blocker treatment was associated with a decrease by almost $60 \%$ in total mortality and hospitalizations for HF. Metoprolol was well tolerated in all patients, irrespective of GFR [112]. The cardiac insufficiency bisoprolol study II (CIBIS-II) was a double-blind, randomized comparison of bisoprolol and placebo in 2647 patients with NYHA classes III and IV HF and a LVEF $<35 \%$. Renal function impairment, defined as a serum creatinine $\geq 3.4 \mathrm{mg} / \mathrm{dL}$ at baseline, was an exclusion criterion. The trial was stopped prematurely, after a mean follow-up of 1.3 years, as bisoprolol 
treatment led to a highly significant reduction in all-cause mortality. In a post hoc analysis, patients were divided into four subgroups according to baseline eGFR $(<45,45-60$, $60-75$, and $>75 \mathrm{~mL} / \mathrm{min}$ per $1.73 \mathrm{~m}^{2}$ ). The beneficial effects of bisoprolol were shown to be similar among all groups. In those with eGFR $<45$, the risk of total mortality or hospitalization for HF was significantly decreased by $28 \%$; however, the rate of bisoprolol discontinuation was higher in this subgroup [113]. SENIORS (Study of the Effects of Nebivolol Intervention on Outcomes and Rehospitalization in Seniors with Heart Failure) investigated the effect of nebivolol versus placebo in 2112 elderly patients with HF (age $>70$ ). The primary outcome (composite of all-cause mortality or CV hospital admission) was significantly reduced in those taking nebivolol (31.1\% versus $35.3 \%$ ). When patients were divided by tertiles of eGFR, nebivolol was found to be similarly effective in all groups. In patients with the lowest eGFR $\left(<55.5 \mathrm{~mL} / \mathrm{min}\right.$ per $\left.1.73 \mathrm{~m}^{2}\right)$, the primary end-point was reduced by $19 \%$, although this did not reach statistical significance. Nebivolol use in these patients was associated with higher rates of drug discontinuation due to bradycardia [114].

There is only one RCT that evaluated the effects of betablocker treatment in dialysis patients with HF. In this 12month study of $114 \mathrm{HD}$ patients with NYHA classes IIIII $\mathrm{HF}$ and $\mathrm{LVEF}<30 \%$, carvedilol therapy was associated with a significant improvement in LVEF and NYHA class, compared to placebo [115]. Subsequent 24-month extended follow-up of the same cohort suggested a survival benefit with carvedilol (51.7\% mortality rate in the carvedilol group versus $73.2 \%$ in the placebo group; $P<0.01)$. There were significantly lower rates of CV mortality $(29.3 \%$ versus $67.9 \%$; $P<0.0001)$ and all-cause hospital admission (34.5\% versus $58.9 \% ; P<0.005)$ in the carvedilol group than in the placebo group. Secondary end-point analyses showed fewer fatal myocardial infarctions, fatal strokes, and hospital admissions for worsening $\mathrm{HF}$ in recipients of carvedilol. A decrease in sudden deaths and pump-failure deaths also was observed, although this did not reach statistical significance. Notably, 2-year echocardiographic data confirmed the significant improvement in LVEF [116].

A recent systematic review and meta-analysis [108] retrieved 6 placebo-controlled trials with beta-blockers, involving 5,972 patients with CKD and systolic HF. The authors found that beta-blocker treatment significantly reduced the risk of all-cause (by 28\%) and CV mortality (by $34 \%$ ), compared to placebo. On the other hand, the risks of bradycardia and hypotension were each increased 5-fold with beta-blocker therapy.

4.2.7. ACE Inhibitors. A subject of many studies, the mechanisms of ACEIs in HF are complex and still not completely understood. By blocking the conversion of angiotensin I to angiotensin II, these drugs promote vasodilation (by reducing the vasoconstrictive effect of angiotensin II) and renal sodium excretion (by decreasing aldosterone release). They inhibit the cardiac RAAS, which is involved in LV hypertrophy and dysfunction. They also block the degradation of bradykinins, thereby stimulating the synthesis of prostaglandins and nitric oxide, which seem to prevent LVH, as well. Other significant effects of ACEIs include the reduction of sympathetic activity, improvement of endothelial function, decrease of proinflammatory cytokines and prothrombotic factors, and stimulation of fibrinolytic factors. All these mechanisms contribute to the amelioration of pulmonary, right ventricular and skeletal muscle function and the increase of arterial compliance [117].

ACEIs have been evaluated in more than 7000 patients with systolic HF, in over 30 placebo-controlled clinical trials. Analyses of these studies showed that these drugs can alleviate symptoms, improve functional status, and reduce the risk of death and hospitalization $[30,32]$. These benefits were seen in patients with various severity and causes of HF [32]. US and European guidelines recommend prescription of ACEIs to all patients with HF due to systolic dysfunction (LVEF $\leq 40 \%$ ), irrespective of symptoms, unless contraindicated or not tolerated $[30,32]$. Patients should not be given an ACEI if they have experienced life-threatening adverse reactions (angioedema or anuric renal failure) during previous exposure to the drug or if they are pregnant. They should take an ACEI with caution if they have very low systemic blood pressure (systolic blood pressure less than $80 \mathrm{~mm} \mathrm{Hg}$ ), markedly increased serum levels of creatinine $(>3 \mathrm{mg} / \mathrm{dL})$, bilateral renal artery stenosis, or elevated levels of serum potassium $(>5.5 \mathrm{mEq} / \mathrm{L})$. Treatment should be initiated at low doses and gradually increased thereafter. The most common adverse effects of ACEIs are hypotension, acute kidney injury, hyperkalemia, and cough [30, 32]. During ACEI therapy, serum creatinine and potassium should be assessed periodically, especially in patients with diabetes and with renal disease $[30,32]$.

The use of ACEIs in patients with CKD and HF seems reasonable, given the well-established simultaneous cardioand renoprotective effects of these drugs [118]. However, there is little evidence that treatment with ACEIs reduces CV morbidity and mortality in this particular population [12]. Furthermore, clinicians are often concerned about the possibly increased risk of adverse reactions from ACEI use in HF patients with impaired kidney function [118].

Experimental studies in animal models of uremia showed that ACEIs are able to prevent LVH and cardiomyocyte loss $[119,120]$, whereas administration of a bradykinin receptor inhibitor completely antagonizes these effects [120], suggesting that the beneficial effects of ACEIs on the CV system may be mediated through bradykinin.

Several observational studies [121] have suggested a favorable impact of ACEIs on survival in patients with CKD and HF. McAlister et al. [121] analyzed data from a prospective cohort of 754 patients with HF and found significant reductions in 1-year mortality with ACEIs and beta-blockers treatments in patients with eGFR $<60 \mathrm{~mL} / \mathrm{min}$, as well as in those with eGFR $\geq 60 \mathrm{~mL} / \mathrm{min}$. A retrospective cohort study of 20,902 hospitalized elderly patients with a LVEF $<40 \%$ [122] showed that, after adjustment for multiple confounders, the prescription of an ACEI on hospital discharge was associated with a significant reduction in mortality; notably, this reduction was greater in patients with serum creatinine 
$>3 \mathrm{mg} / \mathrm{dL}(n=1582)$ than in the rest of the cohort $(37 \%$ versus $16 \%)$. Using propensity scores and multivariable-adjusted Cox regression analyses, Ahmed et al. [123] estimated the effect of ACEIs on 2-year outcomes in 1,707 patients with CKD, taken from the 6,800 patients with systolic HF (LVEF $\leq 45 \%)$ in the Digitalis Investigation Group trial. In this study, CKD was defined as serum creatinine $\geq 1.5 \mathrm{mg} / \mathrm{dL}$ for men and $\geq 1.3 \mathrm{mg} / \mathrm{dL}$ for women. Patients taking ACEIs had significantly lower rates of mortality (hazard ratio $=0.58$ ) and all-cause hospitalizations (hazard ratio $=0.69$ ), compared to those not taking ACEIs.

Moreover, benefits of ACEIs in patients with CKD and HF have been demonstrated by several post hoc analyses of RCTs conducted in the general HF population. The Survival and Ventricular Enlargement (SAVE) study was a randomized trial of captopril versus placebo in 2231 patients with acute myocardial infarction and LVEF $\leq 40 \%$. Patients with serum creatinine $<2.5 \mathrm{mg} / \mathrm{dL}$ were excluded. A secondary analysis of this trial showed that captopril was equally efficacious in subjects with CKD (defined as eGFR $<60 \mathrm{~mL} / \mathrm{min} / 1.73 \mathrm{~m}^{2}$ ) and those without $\mathrm{CKD}$. The relative risk reduction in $\mathrm{CV}$ events and mortality due to captopril was actually higher in subjects with CKD (31\% versus 20\%); however, the interaction between study drug and CKD was not statistically significant [124]. In the studies of left ventricular dysfunction (SOLVD) treatment trial, 2569 ambulatory chronic HF patients with $\mathrm{LVEF} \leq 35 \%$ and serum creatinine $\leq 2.5 \mathrm{mg} / \mathrm{dL}$ were randomized to receive either placebo or enalapril. Of the 2502 patients with baseline serum creatinine data, 1036 had CKD $\left(e G F R<60 \mathrm{~mL} / \mathrm{min} / 1.73 \mathrm{~m}^{2}\right)$. The median follow-up was 35 months. Compared to placebo, enalapril significantly decreased all-cause mortality in non-CKD, but not in CKD, patients (hazard ratio 0.82 versus 0.88 ). However, enalapril did reduce $\mathrm{CV}$ hospitalization in both patients with and patients without CKD (hazard ratio 0.77 versus 0.80 ). Among patients in the enalapril group, serum creatinine elevation was significantly higher in those without CKD (0.09 versus $0.04 \mathrm{mg} / \mathrm{dL}$ ) during the first year of follow-up, but there were no differences in changes in serum potassium (mean increase, $0.2 \mathrm{mEq} / \mathrm{L}$, in both) [125].

In dialysis patients, observational studies have shown that ACEIs can reduce LVH $[126,127]$ and improve survival and CV outcomes [128], and these benefits appeared to be independent of their BP-lowering effect. However, a doubleblind placebo-controlled RCT in 397 HD patients with LVH [129] failed to show any significant effect of ACEI fosinopril on a composite CV end-point. The study was, nevertheless, underpowered to estimate the impact of fosinopril on survival. Chang et al. evaluated the effects of ACEI use among HD patients that participated in the HEMO study [130]. Using proportional hazards regression and a propensity score analysis, the authors found no significant associations between ACEI use and mortality, CV hospitalization, and other CV outcomes. Surprisingly, in the proportional hazards model, ACEI use was even associated with a higher risk of $\mathrm{HF}$ hospitalization. A retrospective analysis of the data from the Minnesota Heart Survey [131] revealed that dialysis patients hospitalized with HF had no benefit from ACEI or ARB treatment, for either short-term (30 days) or long-term (1 year) survival, in striking contrast with all of the other HF patients.

Several concerns exist for the use of ACEIs and ARBs in patients with CKD, particularly about the risk of hyperkalemia and worsening of renal function. However, these effects are usually transient and mild.

A meta-analysis of 5 placebo-controlled RCTs with ACEIs in patients with HF showed that, although the rate of acute kidney injury was higher with ACEIs than with placebo, drug discontinuation was rarely necessary, and renal function returned to baseline in most cases, even without dose adjustment [118, 132]. Furthermore, a systematic review of 12 RCTs with ACEIs for renoprotection in patients with CKD showed that a mild increase in serum creatinine (up to $30 \%$ from baseline) was quite common within the first 2 weeks of therapy; however, this increase was followed by stabilization during the next few weeks $[118,133]$. In patients with both $\mathrm{HF}$ and CKD, a retrospective analysis of the SOLVD studies has shown that the use of ACEIs was associated with a reduction of mortality, even in those with severe renal insufficiency, and did not have an adverse effect on kidney function [134]. Therefore, ACEIs should not be contraindicated in patients with $\mathrm{HF}$ and $\mathrm{CKD}$, and a mild and nonprogressive worsening of renal function at the start of therapy should not be considered, per se, as an indication to discontinue treatment [118]. However, when the GFR falls by $>30 \%$ of the pretreatment baseline, ACEI administration should be halted. Patients should then be evaluated for conditions causing renal hypoperfusion, such as volume depletion (e.g., from diuretics), renal vasoconstriction (e.g., induced by NSAIDs), and severe bilateral renal artery stenosis or stenosis in a single kidney. Unless renovascular disease is found, ACEI therapy can be resumed after correction of the underlying cause of renal ischemia and resolution of the acute kidney injury episode [118]. Reducing the daily diuretic and/or ACEI dose may prevent future worsening of the renal function [74]. It is generally recommended to begin at $15 \%$ to $25 \%$ of the goal dose and, based upon changes in BP and GFR, to increase every 4 to 8 weeks by $25 \%$ to $50 \%$ until the target dose or the highest tolerated dose is reached [135].

The risk of hyperkalemia associated with the use of ACEIs is also a source of concern. In a retrospective analysis of the SOLVD trials, in patients with HF treated with enalapril the incidence of hyperkalemia $\geq 5.5 \mathrm{mEq} / \mathrm{L}$ was $6 \%$, overall; it was higher than in the placebo group and it increased progressively with the severity of the renal dysfunction [118, 136]. Careful monitoring of serum potassium is warranted in all patients with GFR $<60 \mathrm{~mL} / \mathrm{min}$ undergoing ACEI therapy. Concurrent use of other potentially hyperkalemia-inducing drugs, such as NSAIDs, ARBs, and potassium-sparing diuretics, should be avoided or minimized, if possible. A low potassium diet, as well as sodium bicarbonate administration in patients with metabolic acidosis, is also indicated [137]. A potassium level over $5.5 \mathrm{mEq} / \mathrm{L}$ should prompt a reduction in the ACEI dose. If the potassium concentration remains high despite the above measures, the ACEI should be discontinued $[118,137]$. In patients with severe renal impairment, ACEIs 
should always be used with caution, because of their potential risk for adverse events.

4.2.8. Angiotensin II Receptor Blockers. Experience with ARBs in HF trials is much smaller than that with ACEIs. However, several studies showed that ARBs produce hemodynamic, neurohormonal, and clinical effects similar to ACEIs. The ARBs valsartan and candesartan were associated with a reduction in hospitalizations and mortality in two HF RCTs [138, 139]. Given the existing evidence, ACEIs are currently recommended as the first choice for RAAS inhibition in HF, but ARBs are a reasonable alternative, especially for patients who cannot tolerate ACEIs because of cough or angioedema [32]. Side effects like hypotension, worsening renal function, and hyperkalemia are as common as for ACEIs. Therefore, caution is required, by starting treatment at very low doses, followed by slow, step-bystep increases. Additionally, BP, renal function, and serum potassium should be closely monitored.

The dual blockade of the RAAS for the treatment of HF, using a combination of an ACEI with an ARB, seems a reasonable approach. It was shown to reduce the LV size more than either agent alone [140]. However, the clinical benefits of this combination are uncertain. A trial in patients with HF postmyocardial infarction showed that combined therapy did not improve outcomes and resulted in more side effects, compared to each of the two drugs [141]. The addition of ARBs to chronic ACEI therapy caused a modest decrease in hospitalization in 2 studies, with a trend to decreased total mortality in one and no impact on mortality in another $[32,139,140,142]$. Furthermore, the ACC/AHA guidelines suggest that this combination increases the risks of adverse effects [32].

In a study of patients with diabetic nephropathy and CKD stages 3-4, ARBs decreased the risk of developing HF [143]. In a post hoc analysis of the Telmisartan Randomized Assessment Study in ACE Intolerant Subjects with Cardiovascular Disease (TRANSCEND) and the Ongoing Telmisartan Alone and in Combination with Ramipril Global Endpoint Trial (ONTARGET), Tobe et al. [144] examined renal and $\mathrm{CV}$ outcomes in renal subgroups, defined by GFR and albuminuria. The main $\mathrm{CV}$ outcome was the composite of CV death, myocardial infarction, stroke, or hospitalization for HF. The authors found no CV benefit in any subgroup either with telmisartan versus placebo or with dual therapy (telmisartan plus ramipril) versus monotherapy.

Trials of ARBs in patients with HF and CKD are very scarce. In a recent cohort study of 1665 elderly patients with systolic $\mathrm{HF}(\mathrm{LVEF}<45 \%)$ and $\mathrm{eGFR}<60 \mathrm{~mL} / \mathrm{min} / 1.73 \mathrm{~m}^{2}$, followed up for 8 years, Ahmed et al. [145], using a propensity score analysis, found that treatment with ACEIs or ARBs was associated with a significant, but modest, reduction in allcause mortality (hazard ratio 0.86 ; $95 \%$ confidence interval 0.74-0.996; $P=0.045$ ) and no change in hospitalization for HF. A single RCT has been conducted so far using ARBs in ESRD patients. This multicenter Italian trial [146] included $332 \mathrm{HD}$ patients with HF (NYHA II-III; LVEF $\leq 40 \%$ ), who were randomized to telmisartan or placebo, in addition to ACEI therapy. At 3 years, telmisartan significantly reduced all-cause mortality ( $35.1 \%$ versus $54.4 \%$; $P<0.001)$, CV death (30.3\% versus $43.7 \% ; P<0.001)$, and hospital admission for HF (33.9\% versus 55.1\%; $P<$ $0.0001)$. Adverse effects, mainly hypotension, occurred in $16.3 \%$ of the telmisartan group versus $10.7 \%$ in the placebo group.

4.2.9. Aldosterone Antagonists. RAAS inhibition with ACEIs and/or ARBs may not be able to maintain adequate suppression of aldosterone production during long-term therapy, because both aldosterone and angiotensin II ultimately can escape the effects of these drugs, resulting in rebound of aldosterone levels $[147,148]$. This may be a significant issue in patients with HF, since experimental studies suggest that aldosterone has deleterious effects on the structure and function of the heart, independently of and in addition to those of angiotensin II [32]. Aldosterone stimulates sodium and fluid retention and promotes myocardial remodeling and fibrosis, as well as endothelial dysfunction and atherosclerosis $[149,150]$. Aldosterone antagonists (AAs), in addition to ACEIs or ARBs, can provide more complete inhibition of the RAAS, with long-term benefits. However, a higher risk of adverse effects like hyperkalemia and worsening renal function is also to be expected.

Spironolactone and eplerenone were associated with significant reductions in mortality and CV events in patients with systolic HF in the RALES (Randomized Aldactone Evaluation Study) [151] and EPHESUS (Eplerenone Post-Acute Myocardial Infarction Heart Failure Efficacy and Survival) trials $[152,153]$, respectively. On the other hand, many studies have reported an increased incidence of severe hyperkalemia in HF patients treated with AAs in association with ACEIs [118]. Based on these data, US [32] and European [30] guidelines recommend the addition of an AA to an ACEI or an ARB in selected patients with systolic HF (NYHA classes IIIIV, LVEF $<35 \%$ ), but without severe renal dysfunction (serum creatinine $\leq 2.5 \mathrm{mg} / \mathrm{dL}$ in men and $\leq 2.0 / \mathrm{dL}$ in women) and with serum potassium $<5.0 \mathrm{mEq} / \mathrm{L}$. Treatment should be initiated at low doses (e.g., 12.5 or $25 \mathrm{mg}$ of spironolactone or eplerenone), followed by a gradual increase (up to a target of $50 \mathrm{mg}$, if tolerated), under careful surveillance of creatinine and potassium levels. Hyperkalemia and/or worsening of the renal function require dose reduction or even withdrawal of AAs. In men, breast tenderness or enlargement may also occur with spironolactone therapy, in which case switching to eplerenone is indicated. The use of AAs should be avoided whenever adequate monitoring of potassium and creatinine levels is deemed not feasible. Furthermore, AAs are contraindicated in association with other potassiumsparing diuretics, with potassium supplements, and with combinations of ACEIs and ARBs [30, 32].

The effects of AAs on clinical outcomes in patients with HF and moderate or severe CKD are not clear, since both RALES and EPHESUS trials excluded patients with serum creatinine levels $>2.5 \mathrm{mg} / \mathrm{dL}$. A prospective RCT in 
112 patients with stages 2 and 3 CKD showed a significant improvement in LV mass and arterial stiffness with spironolactone versus placebo, independently of central and peripheral BP changes [154]. In Iran, Taheri et al. conducted a small double-blind RCT of spironolactone $25 \mathrm{mg} /$ day versus placebo, in addition to an ACEI or an ARB, in $16 \mathrm{HD}$ patients with HF (NYHA classes III-IV and LVEF < 45\%). After 6 months of treatment, the mean LVEF increased significantly more in the spironolactone group than in the placebo group and the mean LV mass decreased in the spironolactone group, while it increased significantly in the placebo group. The incidence of hyperkalemia was unchanged in both groups [155]. The same research team performed a study with an identical design in $18 \mathrm{PD}$ patients with HF. They found a significant increase in LVEF in the spironolactone group but not in the placebo group and a nonsignificant increase in serum potassium in both groups [156].

The risk of AA-induced hyperkalemia in patients with advanced CKD has rarely been assessed in prospective studies, but most experts believe that this risk is unacceptably high and may become life-threatening, therefore prohibiting the use of these drugs in patients with severe and endstage kidney disease. However, it has been suggested that hyperkalemia may be a less serious issue in HD patients, due to the effective removal of potassium through dialysis, as well as to the ability of these patients to tolerate relatively high levels of potassium without clinical manifestations [157]. Chua et al. recently reviewed 6 RCTs that evaluated the safety of low-dose spironolactone in HD patients (of which, about $50 \%$ were already on ACEI or ARB therapy). The authors found that the incidence of hyperkalemia with spironolactone treatment was similar to that in control groups; however, all these studies involved small populations of compliant subjects, who were at low risk for hyperkalemia [157].

Large-scale RCTs are required to evaluate the efficacy and safety of AAs in addition to ACEIs or ARBs as a treatment strategy for $\mathrm{HF}$ in $\mathrm{CKD}$ patients. In stage $3 \mathrm{CKD}$ patients with $\mathrm{HF}$, AAs may be considered but should be used with great caution, limiting the dose to $25 \mathrm{mg}$ /day, or every other day, and closely monitoring the potassium levels. The AAs should be avoided in patients with CKD stages 4 and 5 [118], although potassium removal by dialysis may lessen the risk of hyperkalemia in patients on renal replacement therapy. The combined use of all three RAAS inhibitors (ACEIs, ARBs, and AAs) cannot be recommended in HF patients, with or without CKD [32].

4.2.10. Digitalis Glycosides. In HF, digitalis glycosides act by inhibiting the Na-K-ATP-ase in the myocardium, thereby increasing cardiac contractility. More recently, these drugs were shown to inhibit Na-K-ATP-ase in noncardiac tissues, as well. In the vagal fibers, this enzymatic inhibition results in a decrease of central nervous system sympathetic activity, whereas in the kidneys it leads to a reduction of renal sodium reabsorption, with subsequent suppression of the RAAS. It has in fact been suggested that these neurohormonal effects of digitalis may be even more important than its myocardial inotropic effect, in terms of clinical benefits for patients with HF [32].

Several small RCTs have shown that short-term treatment with digoxin can improve symptoms, quality of life, and exercise tolerance in patients with HF. These favorable outcomes have been seen both in patients with normal sinus rhythm and in those with atrial fibrillation. In the randomized placebo-controlled Digitalis Investigation Group (DIG) trial [158], in $6800 \mathrm{HF}$ patients with LVEF $\leq 45 \%$ and NYHA classes II-IV, already receiving an ACEI, treatment with digoxin for 2 to 5 years reduced hospitalizations but had no effect on overall mortality. The outcomes did not vary significantly in relation to baseline GFR [159]. However, a post hoc analysis of this trial [160] showed that the effects of digoxin on mortality were dependent on its serum concentrations: at 0.5 to $0.9 \mathrm{ng} / \mathrm{mL}$, digoxin significantly reduced mortality in all HF patients, including those with preserved systolic function, whereas, at higher concentrations, it had no effect in this regard. Low serum digoxin concentration was associated with reduced mortality in most subgroups, including CKD (GFR $<60 \mathrm{~mL} / \mathrm{min} / 1.73 \mathrm{~m}^{2}$ ). In the DIG trial, however, individuals with creatinine levels $>3.0 \mathrm{mg} / \mathrm{dL}$ were not eligible, and only $3 \%$ of participants had a GFR $<30 \mathrm{~mL} / \mathrm{min} / 1.73 \mathrm{~m}^{2}$ [159].

US and European guidelines recommend considering the addition of digoxin in patients with HF who are already treated with optimal doses of diuretics, ACEIs (or ARBs), and beta-blockers and who still have symptoms of HF. Alternatively, an AA could be tried in such cases and digoxin would further be indicated only for those who do not respond or who cannot tolerate AAs. In patients with symptomatic $\mathrm{HF}$ and atrial fibrillation, digoxin can be used to control the heart rate, in addition to, or prior to, a beta-blocker. A daily dose of digoxin of $0.25 \mathrm{mg}$ is most often employed in patients with normal renal function. Potential adverse effects of digitalis include sinoatrial and atrioventricular blocks, atrial and ventricular arrhythmias (especially in the presence of hypokalemia), confusion, nausea, and disturbance of color vision. Digoxin is contraindicated in patients with secondor third-degree heart blocks, suspected sick sinus syndrome, and preexcitation syndromes [32].

Because $85 \%$ of administered digoxin is excreted by the kidneys, the risk of toxicity from this drug is very high in people with CKD [74]. Considering the narrow therapeutic window, the long half-life, and the potential for lethal arrhythmias (especially in the context of HDinduced hypokalemia), most nephrologists generally avoid using digoxin in patients with advanced CKD and ESRD [161]. Chan et al. analyzed the association between digoxin prescription and survival in a retrospective cohort, using covariate- and propensity-score-adjusted Cox models. In over 120,000 incident HD patients, digoxin use was associated with a $28 \%$ increased risk for death. Increasing serum digoxin concentration was also significantly associated with mortality, most markedly in patients with lower predialysis serum potassium [162].

4.2.11. Cardiac Resynchronization Therapy. Dyssynchronous ventricular contraction is often seen in patients with $\mathrm{HF}$ and 
it is usually recognized by the presence of a wide QRS $>0.12 \mathrm{~s}$ on electrocardiogram. Ventricular dyssynchrony results in deficient LV filling, slower rate of increase in LV contractile force, significant mitral regurgitation, paradoxical septal wall motion, and reduced cardiac output $[32,163]$ and has been associated with increased mortality in patients with systolic HF $[164,165]$. Cardiac resynchronization therapy (CRT) employs a biventricular pacemaker device that electrically activates the right and left ventricles in a synchronized manner. Such devices often provide a defibrillator function, as well. Resynchronization therapy can improve ventricular contraction and reduce mitral regurgitation. In a metaanalysis of 14 RCTs that included a total of 4420 patients with moderate or severe HF, systolic dysfunction, and prolonged QRS, in addition to medical treatment, CRT significantly improved LVEF, quality of life, and functional status and decreased hospitalizations by $37 \%$ and all-cause mortality by $22 \%$ [166]. Based on these trials, CRT with defibrillator function is currently recommended for HF patients in NYHA III-IV classes who are symptomatic despite optimal medical therapy and who have a LVEF $\leq 35 \%$ and QRS prolongation $>0.12 \mathrm{~s}[30,32]$. Complications of CRT include lead malfunction or dislodgement, pacemaker problems, and infection.

Most RCTs with resynchronization devices in HF populations had little data on patients with CKD [167]. However, renal subgroup analyses of some of these trials have revealed that the clinical benefits of CRT were similar in all studied patients, irrespective of their baseline GFR. For example, the Multicenter InSync Randomized Clinical Evaluation (MIRACLE) study evaluated CRT in HF patients with NYHA classes III-IV, LVEF $\leq 35 \%$, and QRS $>0.12 \mathrm{~s}$, but with serum creatinine $\leq 3.0 \mathrm{mg} / \mathrm{dL}$. A retrospective analysis of this trial [168] categorized patients according to their baseline eGFR: $\geq 90$ (A), 89 to 60 (B), and 59 to $30(\mathrm{C}) \mathrm{mL} / \mathrm{min} / 1.73 \mathrm{~m}^{2}$. The authors found that, compared to control, CRT improved NYHA class, increased LVEF, and reduced mitral regurgitation in all three eGFR categories, while it improved exercise capacity and decreased LV mass in category C. Furthermore, CRT significantly increased eGFR in category C, suggesting that CRT can indirectly improve renal function, by improving the cardiac function.

Considering the very limited available data, it is difficult to make evidence-based recommendations regarding the use of CRT in CKD patients. Adequate studies are required to evaluate the effect of CRT on morbidity and mortality in this specific population. In HD patients, the transvenous placement of CRT and other cardiac rhythm devices has been associated with an increased risk of device-related infections and central vein stenosis. To avoid such risks, the use of an epicardial approach has recently been suggested for CRT devices in these patients, rather than the classical transvenous route [169].

4.2.12. Optimization of Dialysis. Adequate ultrafiltration is a useful strategy for controlling overhydration and hypertension in dialysis patients. Dietary sodium restriction and the use of low dialysate sodium concentrations are equally important in this regard [12].
In non-ESRD patients with severe, refractory HF, several small studies have demonstrated that ultrafiltration (using either HD or PD techniques) can reduce volume overload, correct hyponatremia, and restore responsiveness to diuretics [30, 32]. In patients with ESRD and HF, the initiation of dialysis may have favorable effects on the heart structure, possibly due to decrease of fluid overload. In a recent retrospective echocardiographic study of 41 patients with advanced CKD and symptomatic HF with low LVEF, Ganda et al. [170] found a significant reduction in LV mass index within a few months from the start of HD, although there was no change in LV shortening fraction.

It has also been suggested that HD might improve cardiac and vascular structure and function in patients with HF, by removal of inflammatory cytokines such as interleukin- 8 and monocyte chemotactic protein-1 [171]. However, the true therapeutic role of this process is unclear, considering that the mass clearance of these cytokines is rather low, that they have a short half-life and can rapidly reappear in plasma, and that beneficial cytokines are lost along with the potentially harmful ones, while the source of inflammation is not affected [89].

On the other hand, HD sessions are often associated with repetitive hemodynamic instability and subsequent myocardial ischemia, resulting in "myocardial stunning" [172]. These short episodes may lead over time to development of permanent regional LV systolic abnormalities and HF [173]. Avoiding large-volume ultrafiltration [69] and improving intradialytic hemodynamics by use of biofeedback mechanisms and cooled dialysate $[174,175]$ might reduce the incidence of such myocardial stunning events. High-flow arteriovenous fistula should also be avoided, as it was shown to contribute to volume overload, high cardiac output, and eccentric LVH [176].

Several studies have suggested that more intensive HD (e.g., short daily or long nocturnal dialysis) has significant CV benefit, compared to conventional thrice-weekly HD [69]. Charra et al. [177] were the first to show that prolonged HD (thrice weekly, 8 hours per session) led to a progressive decrease in volume overload and BP. In a small shortterm randomized trial, Culleton et al. [178] showed marked reductions in $\mathrm{LVH}$ and systolic $\mathrm{BP}$ in patients using frequent nocturnal HD (i.e., 6-8 hours of dialysis treatment at home for 4-6 nights per week), compared to those on conventional HD. Similar results were reported by Ayus et al. [179] in a nonrandomized prospective cohort study of short daily HD versus conventional HD. Chan et al. [180] showed that conversion from conventional to frequent nocturnal HD was associated with a decrease in 24-hour mean BP, peripheral vascular resistance, sympathetic activity, arterial stiffness, and LV mass. In another study, the same authors observed a significant improvement in LVEF in dialysis patients with LV systolic dysfunction, after switching from conventional to frequent nocturnal HD [181]. Finally, the CV benefits of intensive $\mathrm{HD}$ have been confirmed by two very recent RCTs, which compared conventional HD to 6 times per week daily in-center HD (the Frequent Hemodialysis Network Daily Trial) and to 6 times per week nocturnal HD (the FHN Nocturnal Trial), respectively. In the Daily Trial, 
frequent HD resulted in a significant reduction in LV mass (especially in patients with baseline LVH), whereas similar trends were noted in the Nocturnal Trial [182]. All of the above data suggest that ESRD patients with HF might derive substantial benefits from using such intensive HD programs rather than conventional ones. This hypothesis should be considered for further trials.

There is evidence that, during the first few years of dialysis therapy, while residual renal function is preserved, PD may provide better fluid and BP control than HD [183-185], probably due to more abundant urine output and continuous, slow ultrafiltration. However, the prevalence of hypertension and overhydration increases after several years on $\mathrm{PD}$, as a result of progressive loss of diuresis and of peritoneal ultrafiltration capacity [186-189]. Peritoneal ultrafiltration failure is associated with volume expansion, hypertension, LVH, and inflammation [190]. Nevertheless, the use of hypertonic PD solutions, together with salt intake restriction, can maintain adequate BP control and prevent $\mathrm{LVH}$, despite the reduction of residual renal function in long-term PD patients [191]. Icodextrin-based $\mathrm{PD}$ solution is a promising alternative, as it was shown to significantly reduce volume overload and LV mass, compared with a standard solution [192]. Whether this translates to a long-term survival benefit in $\mathrm{PD}$ patients with $\mathrm{HF}$ warrants further investigation.

To date, there are no RCTs comparing the efficacy of PD versus $H D$ for the management of dialysis patients with $H F$. In a cohort of over 100,000 incident dialysis patients with a history of HF, Stack et al. [193] found that PD was associated with a significantly higher mortality, compared with HD, after 2 years of therapy. On the other hand, Vonesh et al. [194] examined data of almost 400,000 dialysis patients $(11.6 \%$ on PD), followed from dialysis inception up to 3 years, and found that those with baseline HF had similar survival rates on both modalities, except for the subgroup of diabetics aged over 45 years, which had a lower mortality on HD than on PD.

4.2.13. Future Directions. Direct renin inhibitors (DRIs) are a newer class of RAAS inhibitors, acting at the first regulatory step of this hormonal system. Initially used as antihypertensive agents, DRIs have more recently been tested in patients with HF. In the ALOFT (Aliskiren Observation of Heart Failure Treatment) study, which included 302 patients with stable HF, adding the DRI aliskiren to ACEIs or ARBs appeared to be safe and effective in decreasing plasma BNP and urinary aldosterone levels [195]. Two other large trials are underway using aliskiren in HF patients. The ATMOSPHERE (Aliskiren Trial to Minimize Outcomes in Patients with Heart Failure) examines the effect of aliskiren on CV mortality and hospitalization in patients with chronic HF, whereas the ASTRONAUT (Aliskiren Trial on Acute Heart Failure Outcomes) evaluates aliskiren in patients stabilized after acute HF [196]. These studies will shed important light on the role of DRIs in the treatment of HF. However, we should mention here the Aliskiren Trial in Type 2 Diabetes Using CardioRenal Disease Endpoints (ALTITUDE), which compared aliskiren to placebo, in addition to ACEI or ARB therapy in patients with diabetic nephropathy and CV disease. This study was prematurely stopped, because of the lack of any prospects of showing a treatment benefit, as well as due to safety concerns, including renal dysfunction, hyperkalemia, hypotension, and an unexpected excess of strokes. As a consequence, it has been suggested that dual aliskiren and ACEI/ARB therapy should not be used in patients with hypertension and CKD $\left(e G F R<60 \mathrm{~mL} / \mathrm{min} / 1.73 \mathrm{~m}^{2}\right)$ [195].

BAY 94-8862 is a novel, nonsteroidal, mineralocorticoid receptor antagonist with greater selectivity than spironolactone and stronger binding affinity than eplerenone. The MinerAlocorticoid Receptor Antagonist Tolerability Study (ARTS) was a multicentre, randomized, double-blind, placebo-controlled, parallel-group study, aiming to evaluate the safety and tolerability of this new drug in patients with systolic HF and CKD [197]. This study showed that BAY 948862 5-10 mg/day was at least as effective as spironolactone 25 or $50 \mathrm{mg} /$ day in decreasing serum levels of BNP and pro$\mathrm{BNP}$, as well as albuminuria, but it was associated with lower incidence of hyperkalaemia (5.3\% versus $12.7 \% ; P=0.048$ ).

Other innovative therapies have been explored in the general population with $\mathrm{HF}$, but with unconvincing results so far. These include TNF- $\alpha$ inhibitors etanercept and infliximab $[198,199]$, the endothelin antagonist bosentan [200], the combined ACE and neutral endopeptidase inhibitor omapatrilat [201], and tolvaptan, a vasopressin (V2) antagonist [202]. Drugs that reverse myocardial fibrosis and matrix remodeling by antagonizing the TGF- $\beta$ pathway or by blockade of further downstream pathways also are under investigation [203]. Further research is needed to clarify the role of these agents in HF patients, including those with CKD.

\section{Conclusions}

(1) Dietary salt restriction and diuretics are recommended for patients with CKD and HF to control fluid overload and symptoms, although their effects on morbidity and mortality are unknown. Loop diuretics should be used as first-line agents in patients with GFR $<30 \mathrm{~mL} / \mathrm{min} / 1.73 \mathrm{~m}^{2}$.

(2) In patients with CKD and ESRD, anemia has been associated with LV hypertrophy and dilatation. In the absence of specific RCTs, we suggest that in people with CKD and HF anemia should be treated according to the guidelines used in the general CKD population, targeting a serum hemoglobin of 11 to $12 \mathrm{~g} / \mathrm{dL}$.

(3) Hyperphosphatemia, secondary hyperparathyroidism, and vitamin D deficiency have all been associated with LV hypertrophy and dysfunction. Achieving adequate phosphate, calcium, vitamin $\mathrm{D}$, and $\mathrm{PTH}$ levels is a reasonable treatment goal in CKD patients, with or without heart disease, although their benefits for preventing or improving HF in these patients have not been proven so far.

(4) In CKD (as well as in non-CKD) patients with systolic HF, several RCTs have shown that beta-blockers (bisoprolol, metoprolol, and carvedilol) can reduce 
(i) Anemia and CKD-mineral and bone disorder should be treated, using the existing guidelines for the general CKD population.

(ii) Dietary salt restriction, diuretics, and adequate ultrafiltration in dialysis patients are key strategies to control fluid overload and HF symptoms.

(iii) Beta-blockers (bisoprolol, metoprolol, and carvedilol) can reduce mortality and should, therefore, be recommended to all patients, unless contraindicated or not tolerated. Treatment must be started at very low doses and carefully uptitrated and Monitored, to avoid worsening HF, bradycardia, and hypotension.

(iv) ACEIs can reduce mortality and should be indicated to all patients with HF and CKD stages 1-3, unless contraindicated or not tolerated. In those with CKD stages 4 and 5, caution is required, considering that the benefits of ACEIs on survival have not been proven and that there is a higher risk of adverse events.

(v) Alternatively, ARBs can be used, particularly in patients who develop cough or angioedema from ACEIs. Dual therapy with ACEIs and ARBs can be considered in resistant cases.

(vi) When using RAAS inhibitors (particularly dual therapy), careful dose titration and clinical monitoring are required to prevent serious side effects, such as hypotension, hyperkalemia, and acute kidney injury.

(vii) In stage $3 \mathrm{CKD}$ patients, aldosterone antagonists may be tried but should be used with great caution and at very low doses, while closely monitoring potassium levels. They should be avoided in patients with CKD stages 4 and 5 .

(viii) The addition of digoxin may be considered in selected cases with poorly controlled symptoms of HF or with high-ventricular rate atrial fibrillation, in the presence of optimal-dose therapy with diuretics, RAAS inhibitors, and beta-blockers. Using very low doses and monitoring of serum digoxin concentration are required.

Box 1: Treatment of HF in patients with CKD: key messages.

mortality and hospitalization rates. Therefore, a betablocker should be recommended to all such patients, unless contraindicated or not tolerated. Treatment must be started at a very low dose and carefully uptitrated and monitored, in order to avoid worsening HF, bradycardia, and hypotension (particularly in dialysis patients). The role of beta-blockers in patients with CKD and HF with normal LVEF is unknown.

(5) Several post hoc analyses of RCTs conducted in the general HF population have shown a favorable effect of ACEIs on survival in patients with CKD and HF; however, subjects with serum creatinine $>2.5 \mathrm{mg} / \mathrm{dL}$ were excluded from those trials. Considering the wellknown CV and renal benefits, we believe that ACEIs should be indicated to all patients with HF and mildto-moderate CKD (stages 1-3), unless contraindicated or not tolerated. On the other hand, in people with $\mathrm{HF}$ and advanced CKD or ESRD, the benefits of ACEIs on survival have not been proven and, furthermore, there is a higher risk of adverse events. Therefore, caution is required in patients with $\mathrm{HF}$ and severely impaired renal function (CKD stages 4 and 5). As an alternative to ACEIs, ARBs can be used, particularly in patients who develop cough or angioedema from ACEIs. Dual therapy with ACEIs and ARBs can also be considered, especially in resistant cases, although the advantage over monotherapy is still uncertain and the risk of adverse effects is likely increased. When using RAAS inhibitors, careful dose titration and clinical monitoring are required to prevent serious side effects, such as hypotension, hyperkalemia, and acute kidney injury. The role of ACEIs and ARBs in patients with CKD and $\mathrm{HF}$ with normal LVEF is unknown. In stage 3 CKD patients with HF, aldosterone antagonists may be considered but should be used with great caution and at very low doses, while closely monitoring the potassium levels. Aldosterone antagonists should be avoided in patients with CKD stages 4 and 5 .

(6) The use of digoxin has very limited indications and requires great prudence in patients with $\mathrm{HF}$ and CKD. The administration of this drug may be considered in selected cases with poorly controlled symptoms of $\mathrm{HF}$ or with high-ventricular rate atrial fibrillation, in the presence of optimal-dose therapy with diuretics, RAAS inhibitors, and beta-blockers. Monitoring of serum digoxin concentration is required, with a target of 0.5 to $0.9 \mathrm{ng} / \mathrm{mL}$; this is usually achieved by administering the drug at very low doses (e.g., $0.125 \mathrm{mg}$ every other day when GFR is 30 to $60 \mathrm{~mL} / \mathrm{min}$ and less frequently in patients with more severe renal dysfunction).

(7) Considering the very limited evidence, no recommendations can be made regarding cardiac resynchronization therapy in CKD patients with $\mathrm{HF}$.

(8) For ESRD patients with HF, the role of dialysis modality choice (HD versus PD) is unclear, but likely irrelevant. However, in both HD and PD patients, adequate ultrafiltration is crucial for controlling volume overload. In HD, large-volume ultrafiltration should 
be avoided, as it may be associated with myocardial stunning episodes. High-flow arteriovenous fistula should also be avoided, as it could contribute to volume overload, high cardiac output, and eccentric LVH. Intensive HD schedules (e.g., short daily or long nocturnal dialysis) and the use of icodextrin-based PD solutions may result in better fluid status and LVH reduction, but whether this translates into survival benefits for HF patients is still unknown.

These conclusions are summarized in Box 1.

\section{Conflict of Interests}

The authors declare that there is no conflict of interests regarding the publication of this paper.

\section{References}

[1] U.S. Renal Data System (USRDS), "Chronic kidney disease in the adult NHANES population," USRDS Annual Report Data, 2009, http://www.usrds.org/2009/pdf/V1_01_09.PDF.

[2] R. N. Foley, P. S. Parfrey, and M. J. Sarnak, "Clinical epidemiology of cardiovascular disease in chronic renal disease," The American Journal of Kidney Diseases, vol. 32, no. 5, supplement 3, pp. S112-S119, 1998.

[3] D. W. Johnson, A. Craven, and N. M. Isbel, "Modification of cardiovascular risk in hemodialysis patients: an evidence-based review," Hemodialysis International, vol. 11, no. 1, pp. 1-14, 2007.

[4] S. M. Bagshaw, D. N. Cruz, N. Aspromonte et al., "Epidemiology of cardio-renal syndromes: workgroup statements from the 7th ADQI consensus conference," Nephrology Dialysis Transplantation, vol. 25, no. 5, pp. 1406-1416, 2010.

[5] A. Kottgen, S. D. Russell, L. R. Loehr et al., "Reduced kidney function as a risk factor for incident heart failure: the atherosclerosis risk in communities (ARIC) study," Journal of the American Society of Nephrology, vol. 18, no. 4, pp. 1307-1315, 2007.

[6] J. M. Soucie and W. M. McClellan, "Early death in dialysis patients: risk factors and impact on incidence and mortality rates," Journal of the American Society of Nephrology, vol. 7, no. 10, pp. 2169-2175, 1996.

[7] J. D. Harnett, R. N. Foley, G. M. Kent, P. E. Barre, D. Murray, and P. S. Parfrey, "Congestive heart failure in dialysis patients: prevalence, incidence, prognosis and risk factors," Kidney International, vol. 47, no. 3, pp. 884-890, 1995.

[8] A. Y. Wang, M. Wang, C. W. Lam, I. H. Chan, S. Lui, and J. E. Sanderson, "Heart failure in long-term peritoneal dialysis patients: a 4-year prospective analysis," Clinical Journal of the American Society of Nephrology, vol. 6, no. 4, pp. 805-812, 2011.

[9] F. C. Trespalacios, A. J. Taylor, L. Y. Agodoa, G. L. Bakris, and K. C. Abbott, "Heart failure as a cause for hospitalization in chronic dialysis patients," The American Journal of Kidney Diseases, vol. 41, no. 6, pp. 1267-1277, 2003.

[10] R. N. Foley, P. S. Parfrey, J. D. Harnett et al., "Clinical and echocardiographic disease in patients starting end-stage renal disease therapy," Kidney International, vol. 47, no. 1, pp. 186-192, 1995.

[11] R. J. Glassock, R. Pecoits-Filho, and S. H. Barberato, "Left ventricular mass in chronic kidney disease and ESRD," Clinical
Journal of the American Society of Nephrology, vol. 4, no. 1, pp. S79-S91, 2009.

[12] C. A. Herzog, R. W. Asinger, A. K. Berger et al., "Cardiovascular disease in chronic kidney disease. A clinical update from kidney disease: improving global outcomes (KDIGO)," Kidney International, vol. 80, no. 6, pp. 572-586, 2011.

[13] G. Cerasola, E. Nardi, A. Palermo, G. Mulè, and S. Cottone, "Epidemiology and pathophysiology of left ventricular abnormalities in chronic kidney disease: a review," Journal of Nephrology, vol. 24, no. 1, pp. 1-10, 2011.

[14] C. Zoccali, F. A. Benedetto, G. Tripepi, and F. Mallamaci, "Cardiac consequences of hypertension in hemodialysis patients," Seminars in Dialysis, vol. 17, no. 4, pp. 299-303, 2004.

[15] B. López, A. González, N. Hermida, C. Laviades, and J. Díez, "Myocardial fibrosis in chronic kidney disease: potential benefits of torasemide," Kidney International, vol. 74, no. 111, pp. S19S23, 2008.

[16] K. Amann, M. Breitbach, E. Ritz, and G. Mall, "Myocyte/capillary mismatch in the heart of uremic patients," Journal of the American Society of Nephrology, vol. 9, no. 6, pp. 1018-1022, 1998.

[17] E. Ritz, "Left ventricular hypertrophy in renal disease: beyond preload and afterload," Kidney International, vol. 75, no. 8, pp. 771-773, 2009.

[18] M. Gross and E. Ritz, "Hypertrophy and fibrosis in the cardiomyopathy of uremia-beyond coronary heart disease," Seminars in Dialysis, vol. 21, no. 4, pp. 308-318, 2008.

[19] E. Ritz and C. Wanner, "The challenge of sudden death in dialysis patients," Clinical Journal of the American Society of Nephrology, vol. 3, no. 3, pp. 920-929, 2008.

[20] R. J. Middleton, P. S. Parfrey, and R. N. Foley, "Left ventricular hypertrophy in the renal patient," Journal of the American Society of Nephrology, vol. 12, no. 5, pp. 1079-1084, 2001.

[21] E. Nardi, A. Palermo, G. Mulè, P. Cusimano, S. Cottone, and G. Cerasola, "Left ventricular hypertrophy and geometry in hypertensive patients with chronic kidney disease," Journal of Hypertension, vol. 27, no. 3, pp. 633-641, 2009.

[22] S. J. Marchais, F. Metivier, A. P. Guerin, and G. M. London, "Association of hyperphosphataemia with haemodynamic disturbances in end-stage renal disease," Nephrology Dialysis Transplantation, vol. 14, no. 9, pp. 2178-2183, 1999.

[23] P. Strózecki, A. Adamowicz, E. Nartowicz, G. OdrowzSypniewska, Z. Włodarczyk, and J. Manitius, "Parathormon, calcium, phosphorus, and left ventricular structure and function in normotensive hemodialysis patients," Renal Failure, vol. 23, no. 1, pp. 115-126, 2001.

[24] C. D. Chue, N. C. Edwards, W. E. Moody, R. P. Steeds, J. N. Townend, and C. J. Ferro, "Serum phosphate is associated with left ventricular mass in patients with chronic kidney disease: a cardiac magnetic resonance study," Heart, vol. 98, no. 3, pp. 219224, 2012.

[25] F. Galetta, A. Cupisti, F. Franzoni et al., "Left ventricular function and calcium phosphate plasma levels in uraemic patients," Journal of Internal Medicine, vol. 258, no. 4, pp. 378384, 2005.

[26] V. Raizada, D. Hillerson, J. S. Amaram, and B. Skipper, "Angiotensin II-mediated left ventricular abnormalities in chronic kidney disease," Journal of Investigative Medicine, vol. 60, no. 5, pp. 785-791, 2012.

[27] S. Steigerwalt, A. Zafar, N. Mesiha, J. Gardin, and R. Provenzano, "Role of aldosterone in left ventricular hypertrophy among African-American patients with end-stage renal disease 
on hemodialysis," The American Journal of Nephrology, vol. 27, no. 2, pp. 159-163, 2007.

[28] G. Grassi, G. Seravalle, L. Ghiadoni et al., "Sympathetic nerve traffic and asymmetric dimethylarginine in chronic kidney disease," Clinical Journal of the American Society of Nephrology, vol. 6, no. 11, pp. 2620-2627, 2011.

[29] National Institute of Clinical Excellence Chronic Heart Failure (NICE), National Clinical Guideline for Diagnosis and Management in Primary and Secondary Care, Royal College of Physicians, London, UK, 2003, http://guidance.nice.org.uk/CG5.

[30] K. Dickstein, A. Cohen-Solal, G. Filippatos et al., "ESC guidelines for the diagnosis and treatment of acute and chronic heart failure 2008," European Heart Journal, vol. 29, no. 19, pp. 2388$2442,2008$.

[31] A. Y. Wang and J. E. Sanderson, "Current perspectives on diagnosis of heart failure in long-term dialysis patients," The American Journal of Kidney Diseases, vol. 57, no. 2, pp. 308-319, 2011.

[32] S. A. Hunt, W. T. Abraham, M. H. Chin et al., "2009 focused update incorporated into the ACC/AHA 2005 guidelines for the diagnosis and management of heart failure in adults: a report of the Aamerican College of Cardiology Foundation/American Heart Association Task Force on practice guidelines: developed in collaboration with the International Society for Heart and Lung Transplantation," Circulation, vol. 119, no. 14, pp. e391e479, 2009.

[33] R. S. Vasan, M. G. Larson, E. J. Benjamin, J. C. Evans, C. K. Reiss, and D. Levy, "Congestive heart failure in subjects with normal versus reduced left ventricular ejection fraction: prevalence and mortality in a population-based cohort," Journal of the American College of Cardiology, vol. 33, no. 7, pp. 19481955, 1999.

[34] T. J. Wang, J. C. Evans, E. J. Benjamin, D. Levy, E. C. LeRoy, and R. S. Vasan, "Natural history of asymptomatic left ventricular systolic dysfunction in the community," Circulation, vol. 108, no. 8, pp. 977-982, 2003.

[35] W. J. Paulus, C. Tschöpe, J. E. Sanderson et al., "How to diagnose diastolic heart failure: a consensus statement on the diagnosis of heart failure with normal left ventricular ejection fraction by the Heart Failure and Echocardiography Associations of the European Society of Cardiology," European Heart Journal, vol. 28, no. 20, pp. 2539-2550, 2007.

[36] R. Pecoits-Filho and S. H. Barberato, "Echocardiography in chronic kidney disease: diagnostic and prognostic implications," Nephron-Clinical Practice, vol. 114, no. 4, pp. c242-c247, 2010.

[37] National Kidney Foundation, "K/DOQI clinical practice guidelines for cardiovascular disease in dialysis patients," The American Journal of Kidney Diseases, vol. 45, no. 4, supplement 3, pp. S1-S153, 2005.

[38] C. Zoccali, F. A. Benedetto, F. Mallamaci et al., "Left ventricular mass monitoring in the follow-up of dialysis patients: prognostic value of left ventricular hypertrophy progression," Kidney International, vol. 65, no. 4, pp. 1492-1498, 2004.

[39] R. N. Foley, P. S. Parfrey, G. M. Kent, J. D. Harnett, D. C. Murray, and P. E. Barre, "Serial change in echocardiographic parameters and cardiac failure in end-stage renal disease," Journal of the American Society of Nephrology, vol. 11, no. 5, pp. 912-916, 2000.

[40] D. Bello, D. J. Shah, G. M. Farah et al., "Gadolinium cardiovascular magnetic resonance predicts reversible myocardial dysfunction and remodeling in patients with heart failure undergoing $\beta$-blocker therapy," Circulation, vol. 108, no. 16, pp. 1945-1953, 2003.

[41] V. Wizemann and M. Schilling, "Dilemma of assessing volume state-the use and the limitations of a clinical score," Nephrology Dialysis Transplantation, vol. 10, no. 11, pp. 2114-2117, 1995.

[42] B. Charra, "Fluid balance, dry weight, and blood pressure in dialysis," Hemodialysis International, vol. 11, no. 1, pp. 21-31, 2007.

[43] K. S. Katzarski, J. Nisell, I. Randmaa, A. Danielsson, U. Freyschuss, and J. Bergstrom, "A critical evaluation of ultrasound measurement of inferior vena cava diameter in assessing dry weight in normotensive and hypertensive hemodialysis patients," The American Journal of Kidney Diseases, vol. 30, no. 4, pp. 459-465, 1997.

[44] S. T. Chang, C. L. Chen, C. C. Chen, F. C. Lin, and D. $\mathrm{Wu}$, "Enhancement of quality of life with adjustment of dry weight by echocardiographic measurement of inferior vena cava diameter in patients undergoing chronic hemodialysis," Nephron-Clinical Practice, vol. 97, no. 3, pp. c90-c97, 2004.

[45] A. Santoro, E. Mancini, C. Basile et al., "Blood volume controlled hemodialysis in hypotension-prone patients: a randomized, multicenter controlled trial," Kidney International, vol. 62, no. 3, pp. 1034-1045, 2002.

[46] A. D. Sinha, R. P. Light, and R. Agarwal, "Relative plasma volume monitoring during hemodialysis aids the assessment of dry weight," Hypertension, vol. 55, no. 2, pp. 305-311, 2010.

[47] J. J. Dasselaar, R. M. Huisman, P. E. de Jong, and C. F. M. Franssen, "Measurement of relative blood volume changes during haemodialysis: merits and limitations," Nephrology Dialysis Transplantation, vol. 20, no. 10, pp. 2043-2049, 2005.

[48] R. Agarwal, K. Kelley, and R. P. Light, "Diagnostic utility of blood volume monitoring in hemodialysis patients," The American Journal of Kidney Diseases, vol. 51, no. 2, pp. 242-254, 2008.

[49] P. Wabel, P. Chamney, U. Moissl, and T. Jirka, "Importance of whole-body bioimpedance spectroscopy for the management of fluid balance," Blood Purification, vol. 27, no. 1, pp. 75-80, 2009.

[50] V. Wizemann, P. Wabel, P. Chamney et al., "The mortality risk of overhydration in haemodialysis patients," Nephrology Dialysis Transplantation, vol. 24, no. 5, pp. 1574-1579, 2009.

[51] P. MacHek, T. Jirka, U. Moissl, P. Chamney, and P. Wabel, "Guided optimization of fluid status in haemodialysis patients," Nephrology Dialysis Transplantation, vol. 25, no. 2, pp. 538-544, 2010.

[52] F. Mallamaci, F. A. Benedetto, R. Tripepi et al., "Detection of pulmonary congestion by chest ultrasound in dialysis patients," JACC: Cardiovascular Imaging, vol. 3, no. 6, pp. 586-594, 2010.

[53] H. Yasue, M. Yoshimura, H. Sumida et al., "Localization and mechanism of secretion of B-type natriuretic peptide in comparison with those of A-type natriuretic peptide in normal subjects and patients with heart failure," Circulation, vol. 90, no. 1, pp. 195-203, 1994.

[54] K. Hosoda, K. Nakao, M. Mukoyama et al., "Expression of brain natriuretic peptide gene in human heart: production in the ventricle," Hypertension, vol. 17, no. 6, part 2, pp. 1152-1155, 1991.

[55] K. Yamamoto, J. C. Burnett Jr., M. Jougasaki et al., "Superiority of brain natriuretic peptide as a hormonal marker of ventricular systolic and diastolic dysfunction and ventricular hypertrophy," Hypertension, vol. 28, no. 6, pp. 988-994, 1996.

[56] A. S. Maisel, P. Krishnaswamy, R. M. Nowak et al., "Rapid measurement of B-type natriuretic peptide in the emergency 
diagnosis of heart failure," The New England Journal of Medicine, vol. 347, no. 3, pp. 161-167, 2002.

[57] P. J. Hunt, A. M. Richards, M. G. Nicholls, T. G. Yandle, R. N. Doughty, and E. A. Espiner, "Immunoreactive amino-terminal pro-brain natriuretic peptide (NT-PROBNP): a new marker of cardiac impairment," Clinical Endocrinology, vol. 47, no. 3, pp. 287-296, 1997.

[58] J. Mant, J. Doust, A. Roalfe et al., "Systematic review and individual patient data meta-analysis of diagnosis of heart failure, with modelling of implications of different diagnostic strategies in primary care," Health Technology Assessment, vol. 13, no. 32, pp. 1-207, 2009.

[59] J. C. Kelder, M. R. Cowie, T. A. McDonagh et al., "Quantifying the added value of BNP in suspected heart failure in general practice: an individual patient data meta-analysis," Heart, vol. 97, no. 12, pp. 959-963, 2011.

[60] C. Balion, P. L. Santaguida, S. Hill et al., "Testing for BNP and NT-proBNP in the diagnosis and prognosis of heart failure," Evidence Report/Technology Assessment, no. 142, pp. 1-147, 2006.

[61] J. L. Januzzi Jr., "The role of natriuretic peptide testing in guiding chronic heart failure management: review of available data and recommendations for use," Archives of Cardiovascular Diseases, vol. 105, no. 1, pp. 40-50, 2012.

[62] M. Codognotto, A. Piccoli, M. Zaninotto et al., "Renal dysfunction is a confounder for plasma natriuretic peptides in detecting heart dysfunction in uremic and idiopathic dilated cardiomyopathies," Clinical Chemistry, vol. 53, no. 12, pp. 20972104, 2007.

[63] Y. Takami, T. Horio, Y. Iwashima et al., "Diagnostic and prognostic value of plasma brain natriuretic peptide in non-dialysisdependent CRF," The American Journal of Kidney Diseases, vol. 44, no. 3, pp. 420-428, 2004.

[64] F. S. Apple, M. A. M. Murakami, L. A. Pearce, and C. A. Herzog, "Multi-biomarker risk stratification of N-terminal proB-type natriuretic peptide, high-sensitivity C-reactive protein, and cardiac troponin $\mathrm{T}$ and I in end-stage renal disease for allcause death," Clinical Chemistry, vol. 50, no. 12, pp. 2279-2285, 2004.

[65] A. Y. Wang, C. W. Lam, C. Yu et al., "N-terminal pro-brain natriuretic peptide: an independent risk predictor of cardiovascular congestion, mortality, and adverse cardiovascular outcomes in chronic peritoneal dialysis patients," Journal of the American Society of Nephrology, vol. 18, no. 1, pp. 321-330, 2007.

[66] F. Mallamaci, C. Zoccali, G. Tripepi et al., "Diagnostic potential of cardiac natriuretic peptides in dialysis patients," Kidney International, vol. 59, no. 4, pp. 1559-1566, 2001.

[67] G. Tripepi, F. Mattace-Raso, F. Mallamaci et al., "Biomarkers of left atrial volume: a longitudinal study in patients with end stage renal disease," Hypertension, vol. 54, no. 4, pp. 818-824, 2009.

[68] A. Y. Wang, "Clinical utility of natriuretic peptides in dialysis patients," Seminars in Dialysis, vol. 25, no. 3, pp. 326-333, 2012.

[69] A. Y. Wang and J. E. Sanderson, "Treatment of heart failure in long-term dialysis patients: a reappraisal," The American Journal of Kidney Diseases, vol. 57, no. 5, pp. 760-772, 2011.

[70] E. Ritz, R. Dikow, M. Adamzcak, and M. Zeier, "Congestive heart failure due to systolic dysfunction: the cinderella of cardiovascular management in dialysis patients," Seminars in Dialysis, vol. 15, no. 3, pp. 135-140, 2002.

[71] M. Özkahya, E. Ok, M. Cirit et al., "Regression of left ventricular hypertrophy in haemodialysis patient by ultrafiltration and reduced salt intake without antihypertensive drugs," Nephrology Dialysis Transplantation, vol. 13, no. 6, pp. 1489-1493, 1998.
[72] A. I. Gunal, I. Karaca, B. Aygen, M. Yavuzkir, A. Dogukan, and H. Celiker, "Strict fluid volume control and left ventricular hypertrophy in hypertensive patients on chronic haemodialysis: a cross-sectional study," Journal of International Medical Research, vol. 32, no. 1, pp. 70-77, 2004.

[73] F. Aslam, A. Haque, J. Haque, and J. Joseph, "Heart failure in subjects with chronic kidney disease: best management practices," World Journal of Cardiology, vol. 2, no. 5, pp. 112-117, 2010.

[74] A. S. Abdo, A. Basu, and S. A. Geraci, "Managing chronic heart failure patient in chronic kidney disease," The American Journal of Medicine, vol. 124, no. 1, pp. 26-28, 2011.

[75] D. M. Mancini, S. D. Katz, C. C. Lang, J. LaManca, A. Hudaihed, and A. Androne, "Effect of erythropoietin on exercise capacity in patients with moderate to severe chronic heart failure," Circulation, vol. 107, no. 2, pp. 294-299, 2003.

[76] D. S. Silverberg, D. Wexler, D. Sheps et al., "The effect of correction of mild anemia in severe, resistant congestive heart failure using subcutaneous erythropoietin and intravenous iron: a randomized controlled study," Journal of the American College of Cardiology, vol. 37, no. 7, pp. 1775-1780, 2001.

[77] D. S. Silverberg, D. Wexler, M. Blum et al., "The use of subcutaneous erythropoietin and intravenous iron for the treatment of the anemia of severe, resistant congestive heart failure improves cardiac and renal function and functional cardiac class, and markedly reduces hospitalizations," Journal of the American College of Cardiology, vol. 35, no. 7, pp. 1737-1744, 2000.

[78] P. van der Meer, H. F. Groenveld, J. L. Januzzi Jr., and D. J. van Veldhuisen, "Erythropoietin treatment in patients with chronic heart failure: a meta-analysis," Heart, vol. 95, no. 16, pp. 13091314, 2009.

[79] A. Levin, C. R. Thompson, J. Ethier et al., "Left ventricular mass index increase in early renal disease: impact of decline in hemoglobin," The American Journal of Kidney Diseases, vol. 34, no. 1, pp. 125-134, 1999.

[80] A. Martinez-Vea, L. Marcas, A. Bardají et al., "Role of oxidative stress in cardiovascular effects of anemia treatment with erythropoietin in predialysis patients with chronic kidney disease," Clinical Nephrology, vol. 77, no. 3, pp. 171-181, 2012.

[81] H. Schmid, H. Schiffl, and S. R. Lederer, "Erythropoiesisstimulating agents, hypertension and left ventricular hypertrophy in the chronic kidney disease patient," Current Opinion in Nephrology and Hypertension, vol. 20, no. 5, pp. 465-470, 2011.

[82] R. N. Foley, P. S. Parfrey, J. Morgan et al., "Effect of hemoglobin levels in hemodialysis patients with asymptomatic cardiomyopathy," Kidney International, vol. 58, no. 3, pp. 1325-1335, 2000.

[83] A. Besarab, W. K. Bolton, J. K. Browne et al., "The effects of normal as compared with low hematocrit values in patients with cardiac disease who are receiving hemodialysis and epoetin," The New England Journal of Medicine, vol. 339, no. 9, pp. 584590, 1998.

[84] T. B. Drüeke, F. Locatelli, N. Clyne et al., "Normalization of hemoglobin level in patients with chronic kidney disease and anemia," The New England Journal of Medicine, vol. 355, no. 20, pp. 2071-2084, 2006.

[85] A. K. Singh, L. Szczech, K. L. Tang et al., "Correction of anemia with epoetin alfa in chronic kidney disease," The New England Journal of Medicine, vol. 355, no. 20, pp. 2085-2098, 2006.

[86] M. A. Pfeffer, E. A. Burdmann, C. Chen et al., "A trial of darbepoetin alfa in type 2 diabetes and chronic kidney disease," The New England Journal of Medicine, vol. 361, no. 21, pp. 20192032, 2009. 
[87] S. A. J. Timmer, K. de Boer, P. Knaapen, M. J. W. Götte, and A. C. van Rossum, "The potential role of erythropoietin in chronic heart failure: from the correction of anemia to improved perfusion and reduced apoptosis?" Journal of Cardiac Failure, vol. 15, no. 4, pp. 353-361, 2009.

[88] F. Locatelli, P. Aljama, B. Canaud et al., "Target haemoglobin to aim for with erythropoiesis-stimulating agents: a position statement by ERBP following publication of the trial to reduce cardiovascular events with aranesp therapy (TREAT) study," Nephrology Dialysis Transplantation, vol. 25, no. 9, pp. 28462850, 2010.

[89] A. Kazory and E. A. Ross, "Contemporary trends in the pharmacological and extracorporeal management of heart failure: a nephrologic perspective," Circulation, vol. 117, no. 7, pp. 975-983, 2008.

[90] S. G. Achinger and J. C. Ayus, "Left ventricular hypertrophy: is hyperphosphatemia among dialysis patients a risk factor?" Journal of the American Society of Nephrology, vol. 17, no. 12, supplement 3, pp. S255-S261, 2006.

[91] Kidney Disease: Improving Global Outcomes (KDIGO) CKDMBD Work Group, "KDIGO clinical practice guideline for the diagnosis, evaluation, prevention, and treatment of chronic kidney disease-mineral and bone disorder (CKD-MBD)," Kidney International Supplements, vol. 76, pp. S1-S2, 2009.

[92] S. D. Navaneethan, S. C. Palmer, M. Vecchio, J. C. Craig, G. J. Elder, and G. F. Strippoli, "Phosphate binders for preventing and treating bone disease in chronic kidney disease patients," Cochrane Database of Systematic Reviews, no. 2, Article ID CD006023, 2011.

[93] J. Silver, M. Rodriguez, and E. Slatopolsky, "FGF23 and PTHdouble agents at the heart of CKD," Nephrology Dialysis Transplantation, vol. 27, no. 5, pp. 1715-1720, 2012.

[94] C. Faul, A. P. Amaral, B. Oskouei et al., "FGF23 induces left ventricular hypertrophy," Journal of Clinical Investigation, vol. 121, no. 11, pp. 4393-4408, 2011.

[95] H. Fujii, J. I. Kim, T. Abe, M. Umezu, and M. Fukagawa, "Relationship between parathyroid hormone and cardiac abnormalities in chronic dialysis patients," Internal Medicine, vol. 46, no. 18, pp. 1507-1512, 2007.

[96] R. E. Weishaar and R. U. Simpson, "Vitamin D3 and cardiovascular function in rats," Journal of Clinical Investigation, vol. 79, no. 6, pp. 1706-1712, 1987.

[97] M. Cozzolino and C. Ronco, "The impact of paricalcitol on left ventricular hypertrophy," Contributions to Nephrology, vol. 171, pp. 161-165, 2011.

[98] J. Wu, M. Garami, T. Cheng, and D. G. Gardner, "1,25(OH)2 vitamin D3, and retinoic acid antagonize endothelin-stimulated hypertrophy of neonatal rat cardiac myocytes," Journal of Clinical Investigation, vol. 97, no. 7, pp. 1577-1588, 1996.

[99] N. Bodyak, J. C. Ayus, S. Achinger et al., "Activated vitamin $\mathrm{D}$ attenuates left ventricular abnormalities induced by dietary sodium in Dahl salt-sensitive animals," Proceedings of the National Academy of Sciences of the United States of America, vol. 104, no. 43, pp. 16810-16815, 2007.

[100] A. Y. Wang, C. W. Lam, J. E. Sanderson et al., "Serum 25-hydroxyvitamin D status and cardiovascular outcomes in chronic peritoneal dialysis patients: a 3-y prospective cohort study," The American Journal of Clinical Nutrition, vol. 87, no. 6, pp. 1631-1638, 2008.

[101] C. W. Park, Y. S. Oh, Y. S. Shin et al., "Intravenous calcitriol regresses myocardial hypertrophy in hemodialysis patients with secondary hyperparathyroidism," The American Journal of Kidney Diseases, vol. 33, no. 1, pp. 73-81, 1999.

[102] P. Ivarsen, J. V. Povlsen, and K. L. Christensen, "Effect of alfacalcidol on cardiac function in patients with chronic kidney disease stage 4 and secondary hyperparathyroidism: a pilot study," Scandinavian Journal of Urology, vol. 46, no. 5, pp. 381388, 2012.

[103] S. Bucharles, S. H. Barberato, A. E. M. Stinghen et al., "Impact of cholecalciferol treatment on biomarkers of inflammation and myocardial structure in hemodialysis patients without hyperparathyroidism," Journal of Renal Nutrition, vol. 22, no. 2, pp. 284-291, 2012.

[104] R. Thadhani, E. Appelbaum, Y. Pritchett et al., "Vitamin D therapy and cardiac structure and function in patients with chronic kidney disease: the PRIMO randomized controlled trial," The Journal of the American Medical Association, vol. 307, no. 7, pp. 674-684, 2012.

[105] G. Ligtenberg, "Regulation of blood pressure in chronic renal failure: determinants of hypertension and dialysis-related hypotension," Netherlands Journal of Medicine, vol. 55, no. 1, pp. 13-18, 1999.

[106] D. Bernardi, L. Bernini, G. Cini, S. Ghione, and I. Bonechi, "Asymmetric septal hypertrophy and sympathetic overactivity in normotensive hemodialyzed patients," The American Heart Journal, vol. 109, no. 3, part 1, pp. 539-545, 1985.

[107] C. Zoccali, F. Mallamaci, S. Parlongo et al., "Plasma norepinephrine predicts survival and incident cardiovascular events in patients with end-stage renal disease," Circulation, vol. 105, no. 11, pp. 1354-1359, 2002.

[108] S. V. Badve, M. A. Roberts, C. M. Hawley et al., "Effects of betaadrenergic antagonists in patients with chronic kidney disease: a systematic review and meta-analysis," Journal of the American College of Cardiology, vol. 58, no. 11, pp. 1152-1161, 2011.

[109] J. Ezekowitz, F. A. McAlister, K. H. Humphries et al., "The association among renal insufficiency, pharmacotherapy, and outcomes in 6,427 patients with heart failure and coronary artery disease," Journal of the American College of Cardiology, vol. 44, no. 8, pp. 1587-1592, 2004.

[110] M. G. Shlipak, W. S. Browner, H. Noguchi, B. Massie, C. D. Frances, and M. McClellan, "Comparison of the effects of angiotensin converting-enzyme inhibitors and beta blockers on survival in elderly patients with reduced left ventricular function after myocardial infarction," The American Journal of Medicine, vol. 110, no. 6, pp. 425-433, 2001.

[111] K. C. Abbott, F. C. Trespalacios, L. Y. Agodoa, A. J. Taylor, and G. L. Bakris, " $\beta$-blocker use in long-term dialysis patients: association with hospitalized heart failure and mortality," Archives of Internal Medicine, vol. 164, no. 22, pp. 2465-2471, 2004.

[112] J. K. Ghali, J. Wikstrand, D. J. van Veldhuisen et al., "The influence of renal function on clinical outcome and response to $\beta$-blockade in systolic heart failure: insights from metoprolol CR/XL randomized intervention trial in chronic HF (MERITHF)," Journal of Cardiac Failure, vol. 15, no. 4, pp. 310-318, 2009.

[113] D. Castagno, P. S. Jhund, J. J. V. McMurray et al., "Improved survival with bisoprolol in patients with heart failure and renal impairment: an analysis of the cardiac insufficiency bisoprolol study II (CIBIS-II) trial," European Journal of Heart Failure, vol. 12, no. 6, pp. 607-616, 2010.

[114] A. Cohen-Solal, D. Kotecha, D. J. van Veldhuisen et al., "Efficacy and safety of nebivolol in elderly heart failure patients with impaired renal function: insights from the SENIORS trial," 
European Journal of Heart Failure, vol. 11, no. 9, pp. 872-880, 2009.

[115] G. Cice, L. Ferrara, A. Di Benedetto et al., "Dilated cardiomyopathy in dialysis patients-beneficial effects of carvedilol: a double-blind, placebo-controlled trial," Journal of the American College of Cardiology, vol. 37, no. 2, pp. 407-411, 2001.

[116] G. Cice, L. Ferrara, A. D’Andrea et al., "Carvedilol increases twoyear survival in dialysis patients with dilated cardiomyopathy: a prospective, placebo-controlled trial," Journal of the American College of Cardiology, vol. 41, no. 9, pp. 1438-1444, 2003.

[117] R. Ascenção, P. Fortuna, I. Reis, and A. V. Carneiro, "Drug therapy for chronic heart failure due to left ventricular systolic dysfunction: a review. III. Angiotensin-converting enzyme inhibitors," Revista Portuguesa de Cardiologia, vol. 27, no. 9, pp. 1169-1187, 2008.

[118] N. Acquarone, C. Castello, G. Antonucci, S. Lione, and P. Bellotti, "Pharmacologic therapy in patients with chronic heart failure and chronic kidney disease: a complex issue," Journal of Cardiovascular Medicine, vol. 10, no. 1, pp. 13-21, 2009.

[119] K. Amann, K. Tyralla, M. Gross et al., "Cardiomyocyte loss in experimental renal failure: prevention by ramipril," Kidney International, vol. 63, no. 5, pp. 1708-1713, 2003.

[120] K. Amann, P. Gassmann, M. Buzello et al., "Effects of ACE inhibition and bradykinin antagonism on cardiovascular changes in uremic rats," Kidney International, vol. 58, no. 1, pp. 153-161, 2000.

[121] F. A. McAlister, J. Ezekowitz, M. Tonelli, and P. W. Armstrong, "Renal insufficiency and heart failure: prognostic and therapeutic implications from a prospective cohort study," Circulation, vol. 109, no. 8, pp. 1004-1009, 2004.

[122] C. D. Frances, H. Noguchi, B. M. Massie, W. S. Browner, and M. McClellan, "Are we inhibited? Renal insufficiency should not preclude the use of ACE inhibitors for patients with myocardial infarction and depressed left ventricular function," Archives of Internal Medicine, vol. 160, no. 17, pp. 2645-2650, 2000.

[123] A. Ahmed, T. E. Love, X. Sui, and M. W. Rich, "Effects of angiotensin-converting enzyme inhibitors in systolic heart failure patients with chronic kidney disease: a propensity score analysis," Journal of Cardiac Failure, vol. 12, no. 7, pp. 499-506, 2006.

[124] M. P. Tokmakova, H. Skali, S. Kenchaiah et al., "Chronic kidney disease, cardiovascular risk, and response to angiotensinconverting enzyme inhibition after myocardial infarction: the survival and ventricular enlargement (SAVE) study," Circulation, vol. 110, no. 24, pp. 3667-3673, 2004.

[125] C. B. Bowling, P. W. Sanders, R. M. Allman et al., "Effects of enalapril in systolic heart failure patients with and without chronic kidney disease: insights from the SOLVD treatment trial," International Journal of Cardiology, vol. 167, no. 1, pp. 151156, 2013.

[126] G. M. London, B. Pannier, A. P. Guerin, S. J. Marchais, M. E. Safar, and J. L. Cuche, "Cardiac hypertrophy, aortic compliance, peripheral resistance, and wave reflection in end-stage renal disease: comparative effects of ACE inhibition and calcium channel blockade," Circulation, vol. 90, no. 6, pp. 2786-2796, 1994.

[127] G. Cannella, E. Paoletti, R. Delfino, G. Peloso, D. Rolla, and S. Molinari, "Prolonged therapy with ACE inhibitors induces a regression of left ventricular hypertrophy of dialyzed uremic patients independently from hypotensive effects," The American Journal of Kidney Diseases, vol. 30, no. 5, pp. 659-664, 1997.
[128] A. P. Guerin, J. Blacher, B. Pannier, S. J. Marchais, M. E. Safar, and G. M. London, "Impact of aortic stiffness attenuation on survival of patients in end-stage renal failure," Circulation, vol. 103, no. 7, pp. 987-992, 2001.

[129] F. Zannad, M. Kessler, P. Lehert et al., "Prevention of cardiovascular events in end-stage renal disease: results of a randomized trial of fosinopril and implications for future studies," Kidney International, vol. 70, no. 7, pp. 1318-1324, 2006.

[130] T. I. Chang, D. Shilane, S. M. Brunelli, A. K. Cheung, G. M. Chertow, and W. C. Winkelmayer, "Angiotensin-converting enzyme inhibitors and cardiovascular outcomes in patients on maintenance hemodialysis," The American Heart Journal, vol. 162, no. 2, pp. 324-330, 2011.

[131] A. K. Berger, S. Duval, C. Manske et al., "Angiotensinconverting enzyme inhibitors and angiotensin receptor blockers in patients with congestive heart failure and chronic kidney disease," The American Heart Journal, vol. 153, no. 6, pp. 10641073, 2007.

[132] M. D. Flather, S. Yusuf, L. Køber et al., "Long-term ACEinhibitor therapy in patients with heart failure or leftventricular dysfunction: a systematic overview of data from individual patients," The Lancet, vol. 355, no. 9215, pp. 1575-1581, 2000.

[133] G. L. Bakris and M. R. Weir, "Angiotensin-converting enzyme inhibitor-associated elevations in serum creatinine: is this a cause for concern?" Archives of Internal Medicine, vol. 160, no. 5, pp. 685-693, 2000.

[134] N. A. Khan, I. Ma, C. R. Thompson et al., "Kidney function and mortality among patients with left ventricular systolic dysfunction," Journal of the American Society of Nephrology, vol. 17, no. 1, pp. 244-253, 2006.

[135] A. H. Gradman and V. Papademetriou, "Combined reninangiotensin-aldosterone system inhibition in patients with chronic heart failure secondary to left ventricular systolic dysfunction," The American Heart Journal, vol. 157, no. 6, supplement, pp. S17-S23, 2009.

[136] S. de Denus, J. Tardif, M. White et al., "Quantification of the risk and predictors of hyperkalemia in patients with left ventricular dysfunction. A retrospective analysis of the studies of left ventricular dysfunction (SOLVD) trials," The American Heart Journal, vol. 152, no. 4, pp. 705-712, 2006.

[137] B. F. Palmer, "Managing hyperkalemia caused by inhibitors of the renin-angiotensin-aldosterone system," The New England Journal of Medicine, vol. 351, no. 6, pp. 585-622, 2004.

[138] C. B. Granger, J. J. V. McMurray, S. Yusuf et al., "Effects of candesartan in patients with chronic heart failure and reduced left-ventricular systolic function intolerant to angiotensinconverting-enzyme inhibitors: the CHARM-alternative trial," The Lancet, vol. 362, no. 9386, pp. 772-776, 2003.

[139] J. N. Cohn and G. Tognoni, "A randomized trial of the angiotensin-receptor blocker valsartan in chronic heart failure," The New England Journal of Medicine, vol. 345, no. 23, pp. 16671675, 2001.

[140] M. Wong, L. Staszewsky, R. Latini et al., "Valsartan benefits left ventricular structure and function in heart failure: Val-HeFT echocardiographic study," Journal of the American College of Cardiology, vol. 40, no. 5, pp. 970-975, 2002.

[141] M. A. Pfeffer, J. J. V. McMurray, E. J. Velazquez et al., "Valsartan, captopril, or both in myocardial infarction complicated by heart failure, left ventricular dysfunction, or both," The New England Journal of Medicine, vol. 349, no. 20, pp. 1893-1906, 2003. 
[142] S. Yusuf, M. A. Pfeffer, K. Swedberg et al., "Effects of candesartan in patients with chronic heart failure and preserved leftventricular ejection fraction: the CHARM-preserved trial," The Lancet, vol. 362, no. 9386, pp. 777-781, 2003.

[143] B. M. Brenner, M. E. Cooper, D. de Zeeuw et al., "Effects of losartan on renal and cardiovascular outcomes in patients with type 2 diabetes and nephropathy," The New England Journal of Medicine, vol. 345, no. 12, pp. 861-869, 2001.

[144] S. W. Tobe, C. M. Clase, P. Gao et al., "Cardiovascular and renal outcomes with telmisartan, ramipril, or both in people at high renal risk: results from the ONTARGET and TRANSCEND studies," Circulation, vol. 123, no. 10, pp. 1098-1107, 2011.

[145] A. Ahmed, G. C. Fonarow, Y. Zhang et al., "Renin-angiotensin inhibition in systolic heart failure and chronic kidney disease," The American Journal of Medicine, vol. 125, no. 4, pp. 399-410, 2012.

[146] G. Cice, A. Di Benedetto, S. D'Isa et al., "Effects of telmisartan added to angiotensin-converting enzyme inhibitors on mortality and morbidity in hemodialysis patients with chronic heart failure: a double-blind, placebo-controlled trial," Journal of the American College of Cardiology, vol. 56, no. 21, pp. 1701-1708, 2010.

[147] J. Staessen, P. Lijnen, R. Fagard, L. J. Verschueren, and A. Amery, "Rise in plasma concentration of aldosterone during long-term angiotensin II suppression," Journal of Endocrinology, vol. 91, no. 3, pp. 457-465, 1981.

[148] C. Borghi, S. Boschi, E. Ambrosioni, G. Melandri, A. Branzi, and B. Magnani, "Evidence of a partial escape of renin-angiotensinaldosterone blockade in patients with acute myocardial infarction treated with ace inhibitors," Journal of Clinical Pharmacology, vol. 33, no. 1, pp. 40-45, 1993.

[149] K. T. Weber, "Aldosterone in congestive heart failure," The New England Journal of Medicine, vol. 345, no. 23, pp. 1689-1697, 2001.

[150] M. Briet and E. L. Schiffrin, "Aldosterone: effects on the kidney and cardiovascular system," Nature Reviews Nephrology, vol. 6, no. 5, pp. 261-273, 2010.

[151] B. Pitt, F. Zannad, W. J. Remme et al., "The effect of spironolactone on morbidity and mortality in patients with severe heart failure," The New England Journal of Medicine, vol. 341, no. 10, pp. 709-717, 1999.

[152] B. Pitt, W. Remme, F. Zannad et al., "Eplerenone, a selective aldosterone blocker, in patients with left ventricular dysfunction after myocardial infarction," The New England Journal of Medicine, vol. 348, no. 14, pp. 1309-1321, 2003.

[153] B. Pitt, H. White, J. Nicolau et al., "Eplerenone reduces mortality 30 days after randomization following acute myocardial infarction in patients with left ventricular systolic dysfunction and heart failure," Journal of the American College of Cardiology, vol. 46, no. 3, pp. 425-431, 2005.

[154] N. C. Edwards, R. P. Steeds, P. M. Stewart, C. J. Ferro, and J. $\mathrm{N}$. Townend, "Effect of spironolactone on left ventricular mass and aortic stiffness in early-stage chronic kidney disease. A randomized controlled trial," Journal of the American College of Cardiology, vol. 54, no. 6, pp. 505-512, 2009.

[155] S. Taheri, M. Mortazavi, S. Shahidi et al., "Spironolactone in chronic hemodialysis patients improves cardiac function," Saudi Journal of Kidney Diseases and Transplantation, vol. 20, no. 3, pp. 392-397, 2009.

[156] S. Taheri, M. Mortazavi, A. Pourmoghadas, S. Seyrafian, Z. Alipour, and S. Karimi, "A prospective double-blind randomized placebo-controlled clinical trial to evaluate the safety and efficacy of spironolactone in patients with advanced congestive heart failure on continuous ambulatory peritoneal dialysis," Saudi Journal of Kidney Diseases and Transplantation, vol. 23, no. 3, pp. 507-512, 2012.

[157] D. Chua, A. Lo, and C. Lo, "Spironolactone use in heart failure patients with end-stage renal disease on hemodialysis: is it safe?” Clinical Cardiology, vol. 33, no. 10, pp. 604-608, 2010.

[158] The Digitalis Investigation Group, "The effect of digoxin on mortality and morbidity in patients with heart failure," The New England Journal of Medicine, vol. 336, no. 8, pp. 525-533, 1997.

[159] M. G. Shlipak, G. L. Smith, S. S. Rathore, B. M. Massie, and H. M. Krumholz, "Renal function, digoxin therapy, and heart failure outcomes: evidence from the digoxin intervention group trial," Journal of the American Society of Nephrology, vol. 15, no. 8, pp. 2195-2203, 2004.

[160] A. Ahmed, M. W. Rich, T. E. Love et al., "Digoxin and reduction in mortality and hospitalization in heart failure: a comprehensive post hoc analysis of the DIG trial," European Heart Journal, vol. 27, no. 2, pp. 178-186, 2006.

[161] D. R. Thiemann, "Digitalis and hemodialysis is a bad combination," Journal of the American Society of Nephrology, vol. 21, no. 9, pp. 1418-1420, 2010.

[162] K. E. Chan, J. M. Lazarus, and R. M. Hakim, "Digoxin associates with mortality in ESRD," Journal of the American Society of Nephrology, vol. 21, no. 9, pp. 1550-1559, 2010.

[163] G. B. Bleeker, J. J. Bax, P. Steendijk, M. J. Schalij, and E. E. van der Wall, "Left ventricular dyssynchrony in patients with heart failure: pathophysiology, diagnosis and treatment," Nature Clinical Practice Cardiovascular Medicine, vol. 3, no. 4, pp. 213219, 2006.

[164] M. Penicka, J. Bartunek, O. Lang et al., "Severe left ventricular dyssynchrony is associated with poor prognosis in patients with moderate systolic heart failure undergoing coronary artery bypass grafting," Journal of the American College of Cardiology, vol. 50, no. 14, pp. 1315-1323, 2007.

[165] S. H. Shin, C. L. Hung, H. Uno et al., "Mechanical dyssynchrony after myocardial infarction in patients with left ventricular dysfunction, heart failure, or both," Circulation, vol. 121, no. 9, pp. 1096-1103, 2010.

[166] F. A. McAlister, J. Ezekowitz, N. Hooton et al., "Cardiac resynchronization therapy for patients with left ventricular systolic dysfunction: a systematic review," The Journal of the American Medical Association, vol. 297, no. 22, pp. 2502-2514, 2007.

[167] L. A. Cannizzaro, J. P. Piccini, U. D. Patel, and A. F. Hernandez, "Device therapy in heart failure patients with chronic kidney disease," Journal of the American College of Cardiology, vol. 58, no. 9, pp. 889-896, 2011.

[168] G. Boerrigter, L. C. Costello-Boerrigter, W. T. Abraham et al., "Cardiac resynchronization therapy improves renal function in human heart failure with reduced glomerular filtration rate," Journal of Cardiac Failure, vol. 14, no. 7, pp. 539-546, 2008.

[169] A. Asif, L. H. Salman, G. G. Lopera, and R. G. Carrillo, "The dilemma of transvenous cardiac rhythm devices in hemodialysis patients: time to consider the epicardial approach," Kidney International, vol. 79, no. 12, pp. 1267-1269, 2011.

[170] A. Ganda, S. D. Weiner, N. L. Chudasama et al., "Echocardiographic changes following hemodialysis initiation in patients with advanced chronic kidney disease and symptomatic heart failure with reduced ejection fraction," Clinical Nephrology, vol. 77, no. 5, pp. 366-375, 2012.

[171] C. Libetta, V. Sepe, M. Zucchi et al., "Intermittent haemodiafiltration in refractory congestive heart failure: BNP and balance 
of inflammatory cytokines," Nephrology Dialysis Transplantation, vol. 22, no. 7, pp. 2013-2019, 2007.

[172] C. W. McIntyre, "Haemodialysis-induced myocardial stunning in chronic kidney disease-a new aspect of cardiovascular disease," Blood Purification, vol. 29, no. 2, pp. 105-110, 2010.

[173] J. O. Burton, H. J. Jefferies, N. M. Selby, and C. W. McIntyre, "Hemodialysis-induced repetitive myocardial injury results in global and segmental reduction in systolic cardiac function," Clinical Journal of the American Society of Nephrology, vol. 4, no. 12, pp. 1925-1931, 2009.

[174] N. M. Selby, S. H. Lambie, P. G. Camici, C. S. Baker, and C. W. McIntyre, "Occurrence of regional left ventricular dysfunction in patients undergoing standard and biofeedback dialysis," The American Journal of Kidney Diseases, vol. 47, no. 5, pp. 830-841, 2006.

[175] N. M. Selby, J. O. Burton, L. J. Chesterton, and C. W. McIntyre, "Dialysis-induced regional left ventricular dysfunction is ameliorated by cooling the dialysate," Clinical Journal of the American Society of Nephrology, vol. 1, no. 6, pp. 1216-1225, 2006.

[176] J. M. MacRae, S. Pandeya, D. P. Humen, N. Krivitski, and R. M. Lindsay, "Arteriovenous fistula-associated high-output cardiac failure: a review of mechanisms," The American Journal of Kidney Diseases, vol. 43, no. 5, pp. e17-e22, 2004.

[177] B. Charra, J. Bergstrom, and B. H. Scribner, "Blood pressure control in dialysis patients: importance of the lag phenomenon," The American Journal of Kidney Diseases, vol. 32, no. 5, pp. 720$724,1998$.

[178] B. F. Culleton, M. Walsh, S. W. Klarenbach et al., "Effect of frequent nocturnal hemodialysis vs conventional hemodialysis on left ventricular mass and quality of life: a randomized controlled trial," The Journal of the American Medical Association, vol. 298, no. 11, pp. 1291-1299, 2007.

[179] J. C. Ayus, M. R. Mizani, S. G. Achinger, R. Thadhani, A. S. Go, and S. Lee, "Effects of short daily versus conventional hemodialysis on left ventricular hypertrophy and inflammatory markers: a prospective, controlled study," Journal of the American Society of Nephrology, vol. 16, no. 9, pp. 2778-2788, 2005.

[180] C. T. Chan, P. J. Harvey, P. Picton, A. Pierratos, J. A. Miller, and J. S. Floras, "Short-term blood pressure, noradrenergic, and vascular effects of nocturnal home hemodialysis," Hypertension, vol. 42, no. 5, pp. 925-931, 2003.

[181] C. Chan, J. S. Floras, J. A. Miller, and A. Pierratos, "Improvement in ejection fraction by nocturnal haemodialysis in end-stage renal failure patients with coexisting heart failure," Nephrology Dialysis Transplantation, vol. 17, no. 8, pp. 1518-1521, 2002.

[182] C. T. Chan, T. Greene, G. M. Chertow et al., "Determinants of left ventricular mass in patients on hemodialysis: frequent hemodialysis network (FHN) trials," Circulation: Cardiovascular Imaging, vol. 5, no. 2, pp. 251-261, 2012.

[183] R. J. Hamburger, P. G. Christ, P. A. Morris, and F. C. Luft, "Hypertension in dialysis patients: does CAPD provide an advantage?" Advances in Peritoneal Dialysis, vol. 5, pp. 91-96, 1989.

[184] N. Lameire, "Cardiovascular risk factors and blood pressure control in continuous ambulatory peritoneal dialysis," Peritoneal Dialysis International, vol. 13, supplement 2, pp. S394S395, 1993.

[185] I. Dasgupta and R. Burden, "Blood pressure control before and after starting dialysis," Nephron-Clinical Practice, vol. 99, no. 3, pp. c86-c91, 2005.
[186] M. K. Menon, D. M. Naimark, J. M. Bargman, S. I. Vas, and D. G. Oreopoulos, "Long-term blood pressure control in a cohort of peritoneal dialysis patients and its association with residual renal function," Nephrology Dialysis Transplantation, vol. 16, no. 11, pp. 2207-2213, 2001.

[187] K. Ateş, G. Nergizoǧlu, K. Keven et al., "Effect of fluid and sodium removal on mortality in peritoneal dialysis patients," Kidney International, vol. 60, no. 2, pp. 767-776, 2001.

[188] G. Enia, F. Mallamaci, F. A. Benedetto et al., "Long-term CAPD patients are volume expanded and display more severe left venticular hypertrophy than haemodialysis patients," Nephrology Dialysis Transplantation, vol. 16, no. 7, pp. 1459-1464, 2001.

[189] C. J. Konings, J. P. Kooman, M. Schonck et al., "Fluid status in CAPD patients is related to peritoneal transport and residual renal function: evidence from a longitudinal study," Nephrology Dialysis Transplantation, vol. 18, no. 4, pp. 797-803, 2003.

[190] P. J. Margetts and D. N. Churchill, "Acquired ultrafiltration dysfunction in peritoneal dialysis patients," Journal of the American Society of Nephrology, vol. 13, no. 11, pp. 2787-2794, 2002.

[191] G. Aşci, M. Özkahya, S. Duman, H. Toz, S. Erten, and E. $\mathrm{Ok}$, "Volume control associated with better cardiac function in long-term peritoneal dialysis patients," Peritoneal Dialysis International, vol. 26, no. 1, pp. 85-88, 2006.

[192] C. J. Konings, J. P. Kooman, M. Schonck et al., "Effect of icodextrin on volume status, blood pressure and echocardiographic parameters: a randomized study," Kidney International, vol. 63, no. 4, pp. 1556-1563, 2003.

[193] A. G. Stack, D. A. Molony, N. S. Rahman, A. Dosekun, and B. Murthy, "Impact of dialysis modality on survival of new ESRD patients with congestive heart failure in the United States," Kidney International, vol. 64, no. 3, pp. 1071-1079, 2003.

[194] E. F. Vonesh, J. J. Snyder, R. N. Foley, and A. J. Collins, "The differential impact of risk factors on mortality in hemodialysis and peritoneal dialysis," Kidney International, vol. 66, no. 6, pp. 2389-2401, 2004.

[195] J. J. V. McMurray, B. Pitt, R. Latini et al., "Effects of the oral direct renin inhibitor aliskiren in patients with symptomatic heart failure," Circulation: Heart Failure, vol. 1, no. 1, pp. 17-24, 2008.

[196] P. S. Sever, A. H. Gradman, and M. Azizi, "Managing cardiovascular and renal risk: the potential of direct renin inhibition," Journal of the Renin-Angiotensin-Aldosterone System, vol. 10, no. 2, pp. 65-76, 2009.

[197] B. Pitt, G. Filippatos, M. Gheorghiade et al., "Rationale and design of ARTS: a randomized, double-blind study of BAY 948862 in patients with chronic heart failure and mild or moderate chronic kidney disease," European Journal of Heart Failure, vol. 14, no. 6, pp. 668-675, 2012.

[198] E. S. Chung, M. Packer, K. H. Lo, A. A. Fasanmade, and J. T. Willerson, "Randomized, double-blind, placebo-controlled, pilot trial of infliximab, a chimeric monoclonal antibody to tumor necrosis factor- $\alpha$, in patients with moderate-to-severe heart failure: results of the anti-TNF therapy against congestive heart failure (ATTACH) trial," Circulation, vol. 107, no. 25, pp. 3133-3140, 2003.

[199] D. L. Mann, J. J. V. McMurray, M. Packer et al., "Targeted anticytokine therapy in patients with chronic heart failure: results of the randomized etanercept worldwide evaluation (RENEWAL)," Circulation, vol. 109, no. 13, pp. 1594-1602, 2004. 
[200] N. F. Kelland and D. J. Webb, "Clinical trials of endothelin antagonists in heart failure: a question of dose?" Experimental Biology and Medicine, vol. 231, no. 6, pp. 696-699, 2006.

[201] M. Packer, R. M. Califf, M. A. Konstam et al., "Comparison of omapatrilat and enalapril in patients with chronic heart failure: the omapatrilat versus enalapril randomized trial of utility in reducing events (OVERTURE)," Circulation, vol. 106, no. 8, pp. 920-926, 2002.

[202] T. Reilly and M. R. Schork, "Vasopressin antagonists: pharmacotherapy for the treatment of heart failure," Annals of Pharmacotherapy, vol. 44, no. 4, pp. 680-687, 2010.

[203] Y. Sata and H. Krum, "The future of pharmacological therapy for heart failure," Circulation Journal, vol. 74, no. 5, pp. 809-817, 2010. 


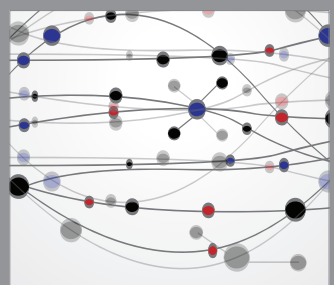

The Scientific World Journal
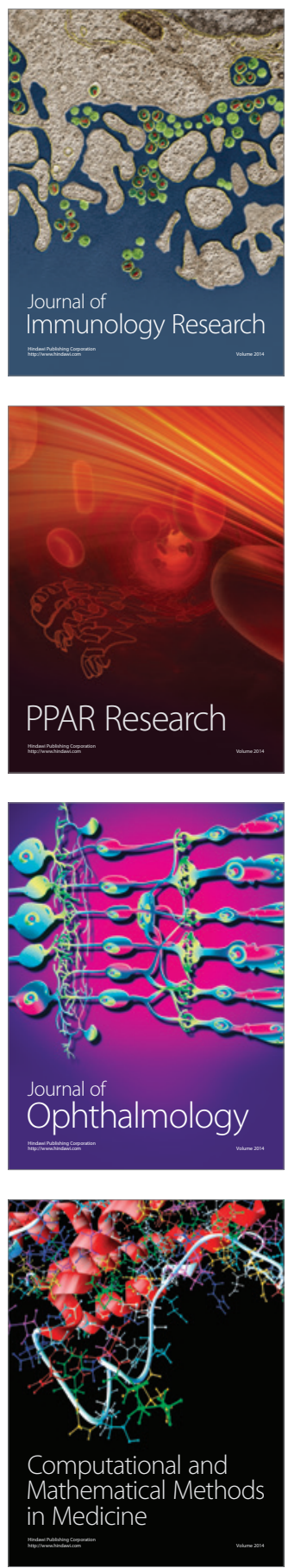

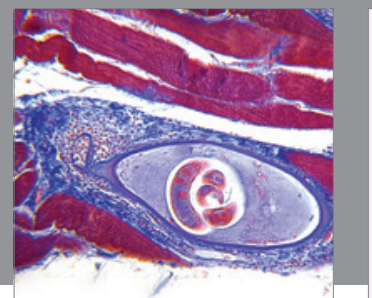

Gastroenterology

Research and Practice
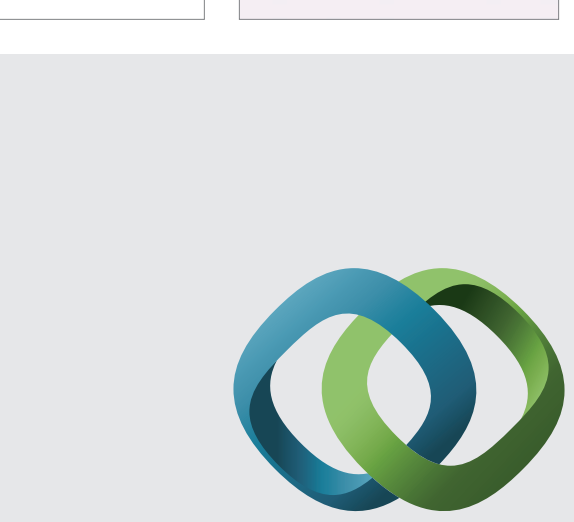

\section{Hindawi}

Submit your manuscripts at

http://www.hindawi.com
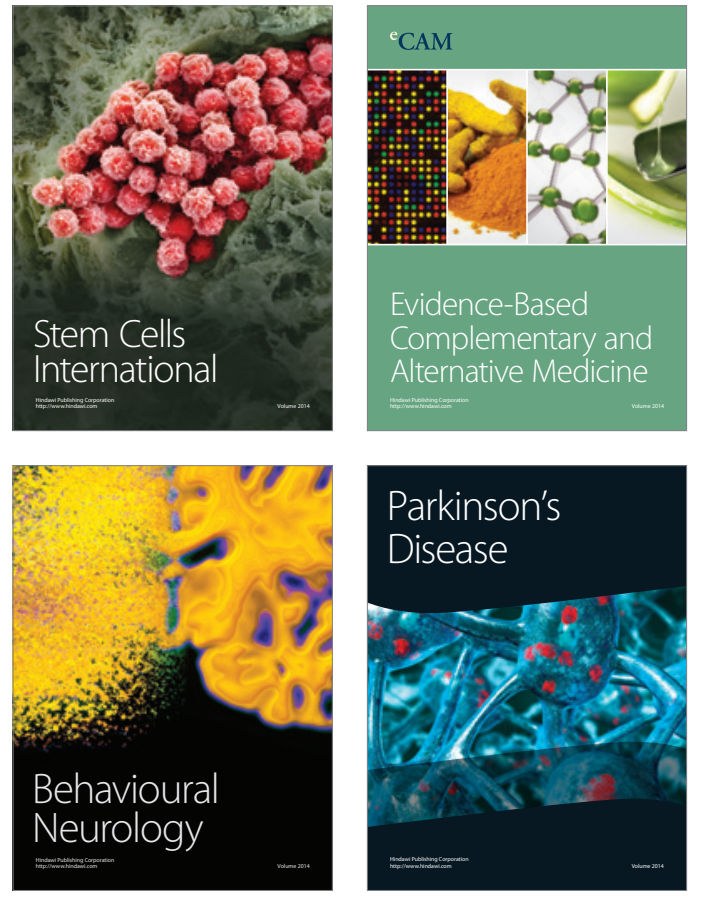
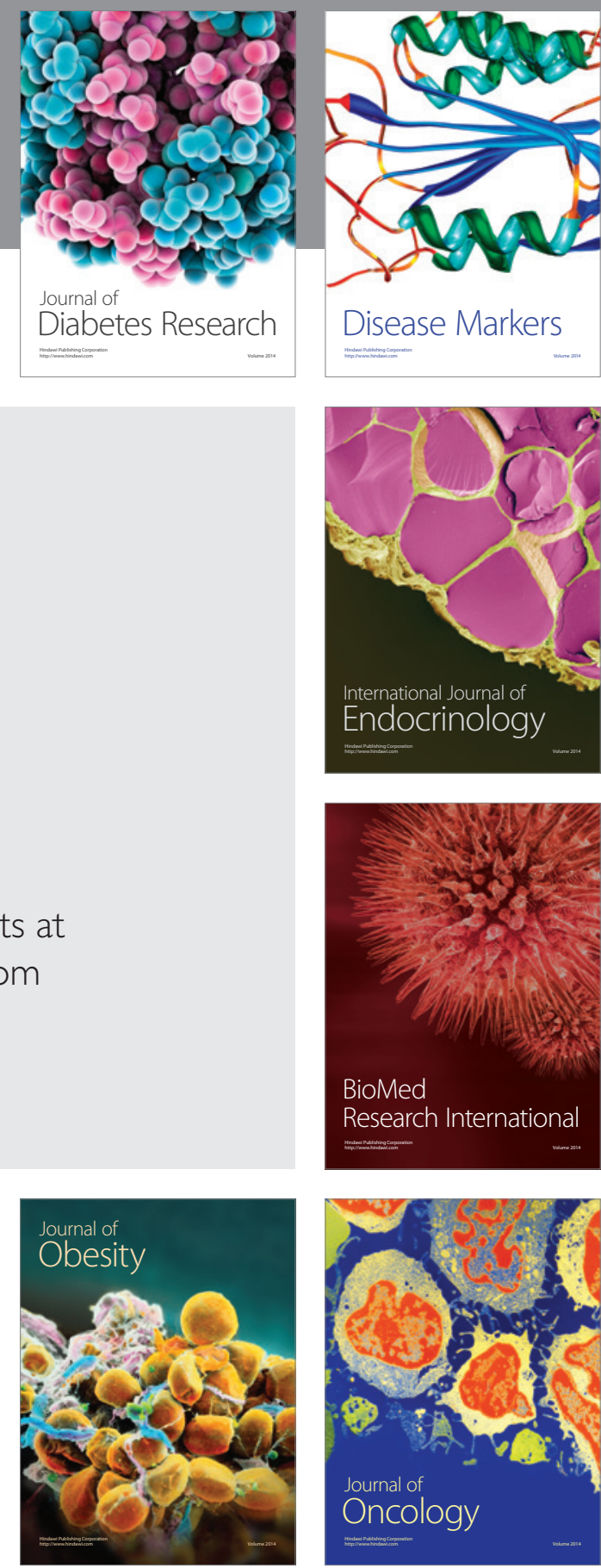

Disease Markers
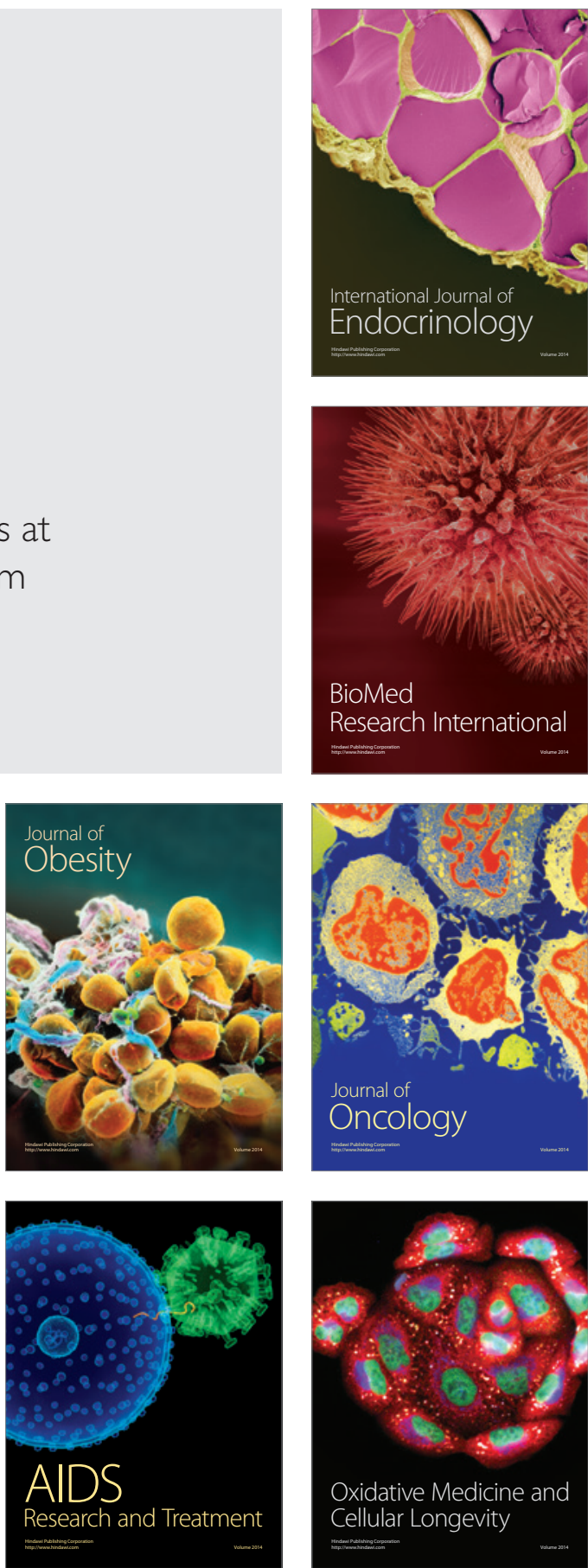